\title{
VLT spectroscopy of globular cluster systems ${ }^{\star}$
}

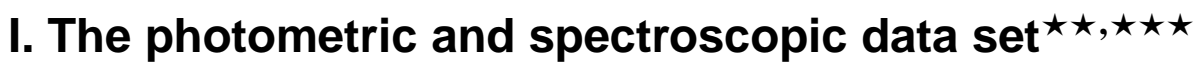

\author{
T. H. Puzia ${ }^{1}$, M. Kissler-Patig ${ }^{2}$, D. Thomas ${ }^{3}$, C. Maraston $^{3}$, R. P. Saglia ${ }^{1}$, \\ R. Bender ${ }^{1,3}$, T. Richtler ${ }^{4}$, P. Goudfrooij ${ }^{5}$, and M. Hempel $^{2}$
}

\author{
1 Sternwarte der Ludwig-Maximilians-Universität, Scheinerstr. 1, 81679 München, Germany \\ e-mail: saglia@usm.uni-muenchen.de \\ 2 European Southern Observatory, 85749 Garching bei München, Germany \\ e-mail: mkissler@eso.org \\ 3 Max-Planck-Institut für extraterrestrische Physik, Giessenbachstrasse, 85748 Garching bei München, Germany \\ e-mail: bender, maraston, dthomas@mpe.mpg.de \\ ${ }^{4}$ Grupo de Astronomía, Departamento de Física, Casilla 160-C, Universidad de Concepción, Concepción, Chile \\ e-mail: tom@coma.cfm.udec.cl \\ 5 Space Telescope Science Institute, 3700 San Martin Drive, Baltimore, MD 21218, USA \\ e-mail: goudfroo@stsci.edu
}

Received 14 May 2003 / Accepted 11 September 2003

\begin{abstract}
We present Lick line-index measurements of extragalactic globular clusters in seven early-type galaxies (NGC 1380, $2434,3115,3379,3585,5846$, and 7192) with different morphological types (E-S0) located in field and group/cluster environments. High-quality spectra were taken with the FORS2 instrument at ESO's Very Large Telescope. 50\% of our data allows an age resolution $\Delta t / t \approx 0.3$ and a metallicity resolution $\sim 0.25-0.4$ dex, depending on the absolute metallicity. Globular cluster candidates are selected from deep $B, V, R, I, K$ FORS2/ISAAC photometry with $80-100 \%$ success rate inside one effective radius. Using combined optical/near-infrared colour-colour diagrams we present a method to efficiently reduce fore-/background contamination down to $\$ 10 \%$. We find clear signs for bi-modality in the globular cluster colour distributions of NGC 1380 , 3115, and 3585. The colour distributions of globular clusters in NGC 2434, 3379, 5846, and 7192 are consistent with a broad single-peak distribution. For the analysed globular cluster systems the slopes of projected radial surface density profiles, of the form $\Sigma(R) \sim R^{-\Gamma}$, vary between $\sim 0.8$ and 2.6. Blue and red globular cluster sub-populations show similar slopes in the clearly bi-modal systems. For galaxies with single-peak globular cluster colour distributions, there is a hint that the blue cluster system seems to have a more extended radial distribution than the red one. Using globular clusters as a tracer population we determine total dynamical masses of host galaxies out to large radii $\left(\sim 1.6-4.8 R_{\text {eff }}\right)$. For the sample we find masses in the range $\sim 8.8 \times 10^{10} M_{\odot}$ up to $\sim 1.2 \times 10^{12} M_{\odot}$. The line index data presented here will be used in accompanying papers of this series to derive ages, metallicities and abundance ratios. A compilation of currently available high-quality Lick index measurements for globular clusters in elliptical, lenticular, and late-type galaxies is provided and will serve to augment the current data set.
\end{abstract}

Key words. galaxies: star clusters - galaxies: general

\section{Introduction}

Compared to their host galaxies globular clusters are remarkably simple stellar structures. They form throughout the

Send offprint requests to: T. H. Puzia,

e-mail: puzia@usm.uni-muenchen.de

* Based on observations collected at the European Southern Observatory, Cerro Paranal, Chile under programme ID P65.N-0281, P66.B-0068, and P67.B-0034.

$\star \star$ Appendix A is only available in electronic form at http://www. edpsciences.org

$\star \star \star$ Appendices B and C are only available in electronic form at the CDS via anonymous ftp to

cdsarc.u-strasbg.fr $(130.79 .128 .5)$ or via

http://cdsweb.u-strasbg.fr/cgi-bin/qcat?J/A+A/415/123 lifetime of the universe and are witnesses of major starformation episodes (e.g. Ashman \& Zepf 1998; Kissler-Patig 2000; Harris 2001). As such, their ages, metallicities, and chemical compositions can provide detailed insights in the formation epochs, processes, and timescales which lead to the assembly of the galaxies we observe in the local universe.

Photometry is one way to assess ages and metallicities of extragalactic globular clusters (among many others Schweizer et al. 1996; Whitmore et al. 1997; Kissler-Patig et al. 1997; Puzia et al. 1999; Maraston et al. 2001; Jordán et al. 2002; Kissler-Patig et al. 2002; Puzia et al. 2002a; Hempel et al. 2003). However, the age-metallicity degeneracy of photometric colours hampers the detailed reconstruction of star-formation histories (e.g. Faber 1972; O’Connell 1976). 
Spectroscopy is an independent alternative to determine star-formation histories and the basic chemistry of globular clusters. The Lick system of absorption line indices (Burstein et al. 1984; Faber et al. 1985; González 1993; Worthey et al. 1994; Worthey \& Ottaviani 1997; Trager et al. 1998), although not free from age-metallicity degeneracy, is a way to measure absorption features which are sensitive to age and metallicity. In combination with state-of-the-art simple stellar population (SSP) models that take the effect of element abundanceratio variations into account (e.g. Maraston et al. 2003; Thomas et al. 2003) indices can shed light on star-formation timescales and the chemical composition. With today's 8-10 m class telescopes the mean Balmer-line index uncertainty for individual extragalactic globular clusters can be reduced to values of the order of the mean isochrone separation in SSP models ( $\sim 0.1 \AA$ between 12 and 13 Gyr). Data of this high-accuracy become available, for the first time, and is in principle capable of resolving star-formation histories even for very old stellar populations.

Previous spectroscopy of globular cluster systems in earlytype galaxies aiming at the derivation of ages and metallicities of single clusters was performed for M 87 (Cohen et al. 1998), NGC 1023 (Larsen \& Brodie 2002), NGC 1316 (Goudfrooij et al. 2001), NGC 1399 (Kissler-Patig et al. 1998; Forbes et al. 2001), NGC 3115 (Kuntschner et al. 2002), NGC 3610 (Strader et al. 2003a), NGC 4365 (Larsen et al. 2003), NGC 4472 (Beasley et al. 2000), and NGC 4594 (Larsen et al. 2002a). However, the data quality allowed only in a few cases to determine the ages and metallicities of individual globular clusters. The data of most studies required summing the spectra of all or at least a given sub-population of clusters to obtain meaningful results. Moreover, the different choices of diagnostic plots (such as $\mathrm{H} \beta$ vs. $\langle\mathrm{Fe}\rangle$ or $\mathrm{H} \gamma$ vs. $\mathrm{Mg} b$ ) made the comparison between galaxies difficult. The existence of SSP models with well-defined abundance ratios only recently allows to account for varying abundance ratios, such as $[\alpha / \mathrm{Fe}]$. Inconsistent use of index passband definitions between data and models introduced additional uncertainties.

In this paper, we present photometry and Lick line-index measurements from our on-going spectroscopic survey of globular cluster systems in early-type galaxies. These high-quality spectroscopic data will be used in subsequent papers of this series (Puzia et al. 2003, in preparation) to derive accurate ages, metallicities, and $[\alpha / \mathrm{Fe}]$ ratios in a self-consistent fashion. The sample presented here includes photometric and spectroscopic data for 143 extragalactic globular clusters.

The present paper is structured as follows: Sect. 2 describes the photometric data set and the candidate selection. Section 3 presents the spectroscopic sample, data reduction, and the selection of candidates. Radial velocity measurements, success rates of the candidate selection, and host galaxy masses are discussed in Sect. 4. Sampled luminosities are discussed in Sect. 5, followed by the description of Lick-index measurements. A compilation of previously published Lick indices for globular clusters in elliptical, lenticular, and late-type galaxies other than studied here is given in Sect. 6. The work is summarised in Sect. 7.

\section{Pre-imaging data}

The host galaxies (NGC 1380, NGC 2434, NGC 3115, NGC 3379, NGC 3585, NGC 5846, and NGC 7192) were selected to sample a significant range in environmental density at intermediate galaxy luminosity and velocity dispersion in the range $-19.2 \gtrsim M_{B} \gtrsim-21.2$ and $184 \lesssim \sigma \lesssim 264 \mathrm{~km} \mathrm{~s}^{-1}$, respectively. All galaxies are of early type $(T<-2)$ according to the RC3 galaxy catalog (de Vaucouleurs et al. 1991). Our sample includes five elliptical and two lenticular galaxies (see Table 1). We used Tully's $\rho_{x y z}$ parameter (Tully 1988) to parameterize the environmental density per $\mathrm{Mpc}^{3}$ of galaxies which are brighter than $M_{B}=-16$ to separate field from group/cluster environment. In this work, we define galaxies with $\rho_{x y z}<0.5$ as field objects and galaxies with $\rho_{x y z}>0.5$ as group/cluster members. Three galaxies of our sample (NGC 1380, NGC 3379, and NGC 5846) are assigned group/cluster membership, while the remaining four galaxies are considered field members. Among other relevant parameters, $\rho_{x y z}$ and $M_{B}$ are summarized in Table 1 for all our sample galaxies.

The imaging mode of FORS2 at ESO's Very Large Telescope was used to obtain pre-imaging data for each galaxy in multiple filters to select candidate globular clusters for spectroscopic follow-up. Exposure times in each filter are summarized in Table 2. Standard calibration routines in IRAF were applied to bias and flatfield the images. Galaxy light was subtracted by, first, removing stellar objects from the image by using SExtractor (v2.1.6 Bertin \& Arnouts 1996) and, second, smoothing the residual image with a large median filter. The median filtered image was subsequently subtracted from the original image. This procedure was iterated with a smaller median filter to discard weak haloes around objects on steep galaxy-light slopes near the central regions. SExtractor was used to perform photometry in a 6-pixel-diameter aperture which was found to yield the highest signal to noise of measured magnitudes. The residual flux which falls outside the 6-pixel aperture was measured in a growth-curve analysis for a handful of objects in each filter for each single galaxy. Uncertainties for the aperture correction were found to be of the order $\sim 0.01 \mathrm{mag}$. All instrumental magnitudes were subsequently corrected with these corrections found.

Each data set was calibrated using standard-star observations for each night provided by the quality control group of ESO. All observations were performed under photometric conditions and could be calibrated to an average intrinsic accuracy of $\sim 0.03$ mag.

We augment our optical photometric data with the recently published near-infrared data for NGC 3115 (Puzia et al. 2002a) and for NGC 5846 and NGC 7192 (Hempel et al. 2003).

\subsection{Consistency check with WFPC2 photometry}

We use WFPC2/HST archive data which were obtained from ST-ECF in Garching to check for consistency of our photometry with that of WFPC2. For the sake of homogeneity, we use the pipeline-processed, co-added (averaged), and cosmic-cleaned image cubes provided as association files by the archive. Photometry was performed in the standard 
Table 1. Basic information on host galaxies. The references are: (1) de Vaucouleurs et al. (1991); (2) NED ${ }^{a}$; (3) Schlegel et al. (1998); (4) Buta \& Williams (1995); (5) Pahre (1999); (6) Tonry et al. (2001); (7) Tully (1988); (8) McElroy (1995); (9) Kissler-Patig et al. (1997); (10) Ashman \& Zepf (1998).

\begin{tabular}{|c|c|c|c|c|c|c|c|c|}
\hline Parameter & NGC 1380 & NGC 2434 & NGC 3115 & NGC 3379 & NGC 3585 & NGC 5846 & NGC 7192 & Ref. \\
\hline type & $-2 / \mathrm{LA}$ & $-5 / \mathrm{E} 0+$ & $-3 / \mathrm{L}-$ & $-5 / \mathrm{E} 1$ & $-5 / \mathrm{E} 6$ & $-5 / \mathrm{E} 0$ & $-4.3 / \mathrm{E}+$ & (1) \\
\hline RA (J2000) & 033627 & 073451 & 100514 & 104750 & 111317 & 150629 & 220650 & (2) \\
\hline $\operatorname{DEC}(\mathrm{J} 2000)$ & -345834 & -691701 & -074307 & +123455 & -264518 & +013621 & -641857 & (2) \\
\hline$v_{\mathrm{rad}}$ & $1841 \pm 15$ & $1390 \pm 27$ & $670 \pm 12$ & $889 \pm 12$ & $1399 \pm 27$ & $1710 \pm 12$ & $2897 \pm 32$ & (1) \\
\hline$E_{B-V}$ & 0.017 & 0.248 & 0.047 & 0.024 & 0.064 & 0.055 & 0.034 & (3) \\
\hline$(B-V)_{\mathrm{eff}, \mathrm{o}}$ & 0.92 & 1.09 & 0.94 & 0.98 & 0.99 & 1.03 & 0.97 & (1) \\
\hline$(V-I)_{\mathrm{eff}, \mathrm{o}}$ & 1.21 & 1.42 & 1.25 & 1.24 & 1.26 & 1.28 & 1.24 & (4) \\
\hline$(V-K)_{\mathrm{eff}, \mathrm{o}}$ & 3.36 & 3.10 & $\ldots$ & 3.08 & $\ldots$ & 3.12 & $\ldots$ & (5) \\
\hline$(m-M)_{V}$ & $31.23 \pm 0.18$ & $31.67 \pm 0.29$ & $29.93 \pm 0.09$ & $30.12 \pm 0.11$ & $31.51 \pm 0.18$ & $31.98 \pm 0.20$ & $32.89 \pm 0.32$ & (6) \\
\hline$M_{B}$ & -20.04 & -19.48 & -19.19 & -19.39 & -20.93 & -21.16 & -20.55 & (7) \\
\hline$a / b^{b}$ & 0.56 & 0.94 & 0.49 & 0.93 & 0.58 & 0.89 & 1.00 & (7) \\
\hline$\sigma^{c}$ & 225 & 204 & 264 & 209 & 218 & 252 & 184 & (8) \\
\hline$\rho_{x y z}^{d}$ & 1.54 & 0.19 & 0.08 & 0.52 & 0.12 & 0.84 & 0.28 & (7) \\
\hline$N_{\mathrm{GC}}^{e}$ & $560 \pm 30$ & $\ldots$ & $520 \pm 120$ & $300 \pm 160$ & $\ldots$ & $2200 \pm 1300$ & $\ldots$ & (9), (10) \\
\hline$S_{\mathrm{N}}^{f}$ & $1.5 \pm 0.5$ & $\ldots$ & $1.6 \pm 0.4$ & $1.2 \pm 0.6$ & $\ldots$ & $3.5 \pm 2.1$ & $\ldots$ & (9), (10) \\
\hline
\end{tabular}

a http://nedwww.ipac. caltech.edu

${ }^{b}$ Ratio of semi-minor/semi-major axis.

c Central velocity dispersion in $\mathrm{km} \mathrm{s}^{-1}$.

${ }^{d}$ Environmental density of galaxies brighter than $M_{B}=-16$ in galaxies/megaparsec ${ }^{3}$ (Tully 1988).

$e$ Total number of globular clusters.

$f$ Specific frequency, $S_{\mathrm{N}}=N_{\mathrm{GC}} \cdot 10^{0.4 \cdot\left(M_{V}+15\right)}$ (Harris \& van den Bergh 1981).

Table 2. Journal of photometric observations. Exposure times are given in seconds.

\begin{tabular}{lccccc}
\hline \hline Galaxy & $B$ & $V$ & $R$ & $I$ & $K$ \\
\hline NGC 1380 & $\ldots$ & 700 & $\ldots$ & 700 & $\ldots$ \\
NGC 2434 & $\ldots$ & 700 & $\ldots$ & 700 & $\ldots$ \\
NGC 3115 & 160 & 300 & 160 & 300 & $15500^{a}$ \\
NGC 3379 & $\ldots$ & 300 & $\ldots$ & 300 & $\ldots$ \\
NGC 3585 & 800 & $\ldots$ & $\ldots$ & 800 & $\ldots$ \\
NGC 5846 & 900 & 300 & 160 & 300 & $10000^{b}$ \\
NGC 7192 & 900 & 600 & 900 & 600 & $12000^{b}$ \\
\hline \\
${ }^{a}$ Data were taken from Puzia et al. (2002a). \\
${ }^{b}$ Data were taken from Hempel et al. (2003).
\end{tabular}

Holtzman et al. (1995a) 0.5" radius aperture using SExtractor and corrected for the y-CTE ramp as described in Holtzman et al. (1995b). We transform the WFPC2 filters F450W, F555W, F702W, and F814W to Johnson-Cousins filters $B, V$, $R$, and $I$, respectively, using the prescriptions in Holtzman et al. (1995a).

At the distance of the two nearest galaxies in our sample, NGC 3115 and NGC 3379 (see Table 1), globular clusters are resolved by HST. Therefore their photometry needs an additional zero-point correction since the standard aperture corrections for stellar profiles (see Holtzman et al. 1995a) do not apply. At the distance of NGC 3115 a globular cluster with a typical half-light radius of $3 \mathrm{pc}$ appears with $\sim 0.06^{\prime \prime}$ on the chip and will be resolved by the planetary camera $\left(0.0455^{\prime \prime} / \mathrm{pix}\right)$.
However, such objects on the wide-field chips (0.0996"/pix) are on the edge of being resolved. $\sim 90 \%$ of Milky Way globular clusters have half-light radii smaller than 3 pc (Harris 1996) and their counterparts in NGC 3115 and NGC 3379 are expected to have similar size distributions (e.g. Kundu \& Whitmore 1998; Larsen et al. 2001). Even if most comparison objects are globular clusters with half-light radii $\sim 3 \mathrm{pc}$, we do not expect the aperture corrections to be larger than the total uncertainty of the FORS2 and WFPC2 photometric calibration. For the remaining sample galaxies most globular clusters are not resolved by WFPC2. Hence, we do not apply any aperture corrections to the WFPC2 photometry.

We find good agreement between the two photometric data sets with offsets $\lessgtr|0.08|$ mag (see Table 3 ) which were calculated in the sense $\Delta m=m_{\mathrm{FORS} 2}-m_{\mathrm{WFPC} 2}$. On average, the offsets are small and of the order of their uncertainties showing no systematics with galaxy distance, background level, etc. Hence, we do not apply these corrections to our FORS2 data.

\subsection{Colour-magnitude diagrams}

In the following two sections we present the photometric selection of globular cluster candidates for follow-up spectroscopy. For this purpose we use colour-magnitude and colour-colour diagrams.

Figure 1 shows colour-magnitude diagrams (CMDs) for our sample globular cluster systems using the FORS2 pre-imaging data. Small dots indicate the entire data extracted from our pre-imaging fields. Asterisks, squares, and 4-prong stars show 

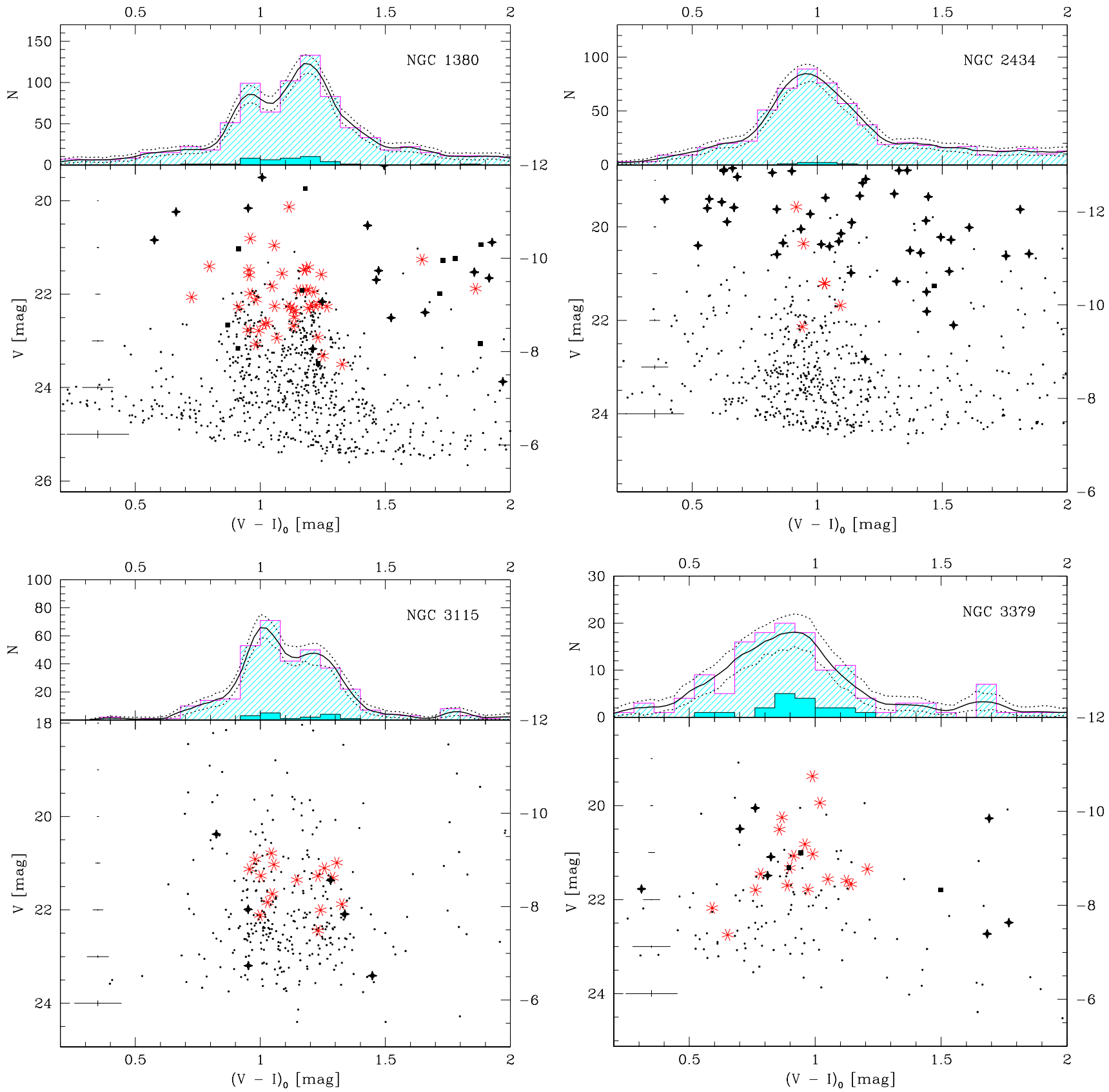

Fig. 1. $V$ vs. $V-I$ CMDs for globular cluster systems of NGC 1380, NGC 2434, NGC 3115, and NGC 3379. Asterisks indicate spectroscopically confirmed globular clusters; 4-prong stars and filled squares show objects whose redshifts are consistent with foreground stars and background galaxies, respectively. Left ordinates show apparent magnitudes, right ordinates indicate absolute magnitudes calculated using distance moduli from Table 1. Each panel shows average photometric error bars near the left ordinate. The upper sub-panels show histograms of the colour distributions. Hatched histograms were created from the entire photometric data, solid histograms show colour distributions of spectroscopically confirmed globular clusters. The solid and dotted lines are probability density estimates with their bootstraped $90 \%$ confidence limits (for details see Silverman 1986). The bin size of the histograms was adjusted to 0.08 mag which roughly corresponds to the mean photometric error.

magnitudes and colours of spectroscopically confirmed globular clusters (see Sect. 4.1), background galaxies, and foreground stars, respectively.

Globular cluster candidates were pre-selected by their photometric error $(\Delta m \leq 0.2 \mathrm{mag}), F W H M(\leq 1.5\langle F W H M\rangle)$, PSF ellipticity $(\epsilon \leq 0.6)$, and the SExtractor star/galaxy classifier $(>0.0$, i.e. only clearly extended sources were rejected). Due to the good spatial coverage of the FORS2 field of view, each sample is the most comprehensive compilation of candidate globular cluster colours so far. We find clear bi-modalities in NGC 1380, NGC 3115, and NGC 3585 (note that we lack $V$ band photometry for NGC 3585 and use $B$ magnitudes instead). Weak indications for possible multi-modality are found in each of the former three colour distributions. To test these distributions for $b i$-modality, we apply the KMM algorithm (Ashman et al. 1994) to the constrained samples with a colour range $0.8<V-I<1.4$ for NGC $1380,0.9<V-I<1.4$ for NGC 3115, and $1.2<B-I<2.4$ for NGC 3585 . 

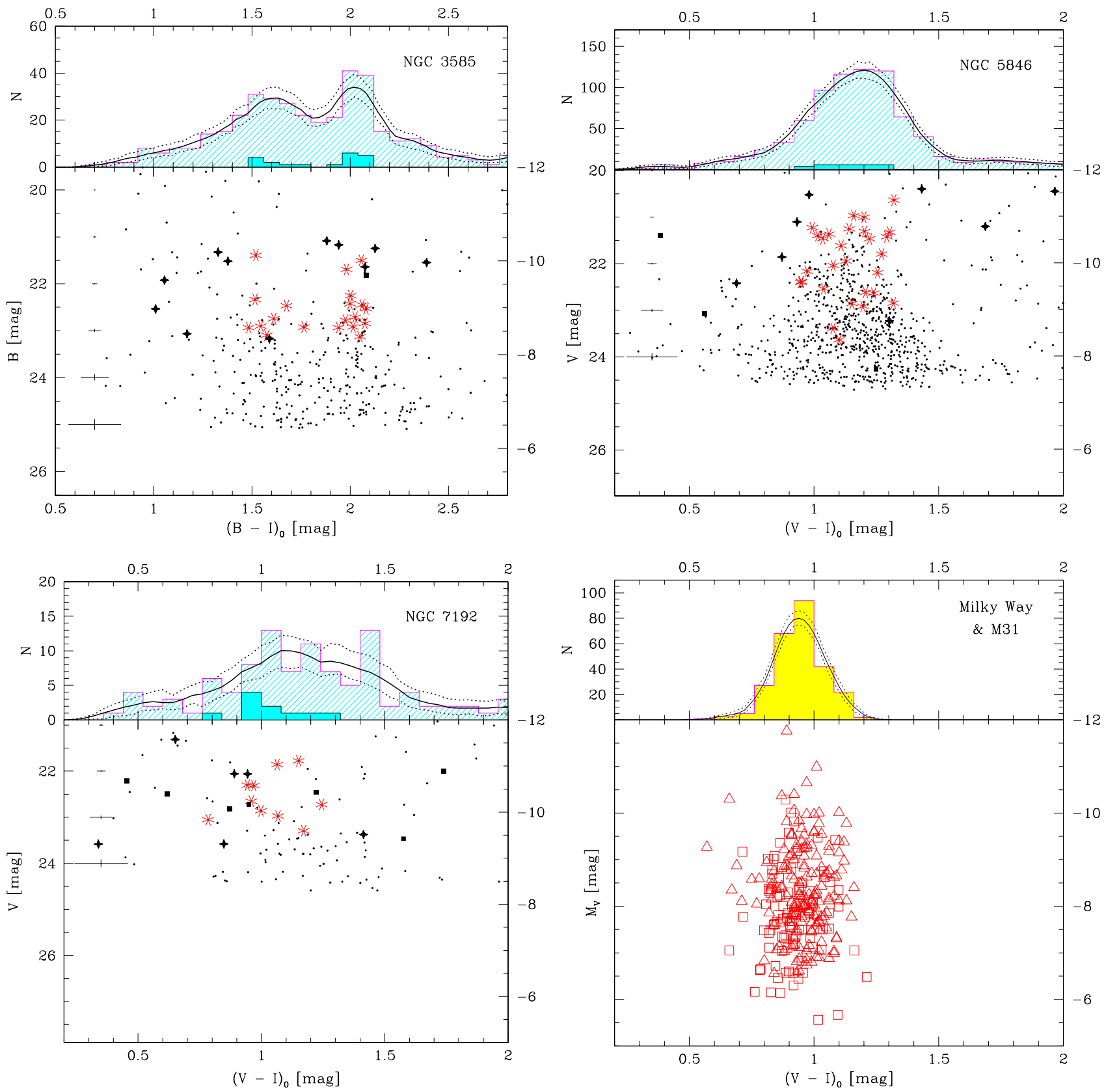

Fig. 1. continued. CMDs for NGC 3585, NGC 5846 and NGC 7192. Please, note that due to the lack of $V$-band data, we plot $B$ vs. $B-I$ for NGC 3585. For comparison, the CMD for globular clusters in the Milky Way (squares) and M 31 (triangles) is plotted in the lower right panel. The Milky Way data were taken from the 1999 update of the McMaster catalog (Harris 1996), while M 31 data are from Barmby et al. (2000).

The code yields peaks at $V-I=0.94 \pm 0.01$ and $1.20 \pm 0.01 \mathrm{mag}$ for NGC 1380 (with a number ratio blue/red $=0.46$ ), $V-I=$ $1.01 \pm 0.01$ and $1.21 \pm 0.01 \mathrm{mag}$ for NGC 3115 (blue $/ \mathrm{red}=$ $0.87)$, and $B-I=1.56 \pm 0.01$ and $2.05 \pm 0.01$ for NGC 3585 (blue/red = 1.02).

The colour histograms for NGC 2434, NGC 3379, NGC 5846, and NGC 7192 are consistent with single-peak distributions. However, it is interesting to note that these peaks are systematically broader than colour peaks of sub-groups in clearly multi-modal distributions. One reason for a broad single-peak colour distribution (in the optical) might be that the gap between two old metal-poor and metal-rich globular cluster populations is filled by metal-rich intermediate-age clusters.
A large spread in age and metallicity in the underlying globular cluster system would naturally produce a sequence in colours rather than multiple distinct peaks. In the context of the hierarchical galaxy formation scenario, clearly bi-modal globular cluster colour distributions may have to be considered as special cases of a wide range of colour distribution morphologies.

Indeed, using a combination of optical and near-infrared photometry it was recently shown that NGC 4365 hosts an intermediate-age globular cluster sub-population which produces a single-peak $V-I$ colour distribution (Puzia et al. 2002a). Hempel et al. (2003) find intermediate-age globular clusters in NGC 5846 and NGC 7192, although the case of NGC 7192 is less conclusive. For details on 
Table 3. Photometric offsets between FORS 2 and WFPC 2 data. Offsets are defined in the sense $\Delta m=m_{\mathrm{FORS} 2}-m_{\mathrm{WFPC} 2}$. The given uncertainties are the errors of the mean. The last column shows the number of objects from which the photometric offsets were calculated.

\begin{tabular}{lccccc}
\hline \hline Galaxy & $\Delta B$ & $\Delta V$ & $\Delta R$ & $\Delta I$ & $N_{\text {obj }}$ \\
\hline NGC 1380 & $\ldots$ & -0.035 & $\ldots$ & $\ldots$ & 142 \\
& & \pm 0.018 & & & \\
NGC 2434 & $\ldots$ & -0.012 & $\ldots$ & -0.044 & 113 \\
& & \pm 0.021 & & \pm 0.022 & \\
NGC 3115 & $\ldots$ & +0.078 & $\ldots$ & +0.043 & 79 \\
& & \pm 0.017 & & \pm 0.017 & \\
NGC 3379 & $\ldots$ & -0.005 & $\ldots$ & +0.079 & 46 \\
& & \pm 0.020 & & \pm 0.030 & \\
NGC 3585 & $\ldots$ & $\ldots$ & $\ldots$ & -0.080 & 89 \\
& & & & \pm 0.025 & \\
NGC 5846 & $\ldots$ & +0.011 & +0.066 & -0.055 & 89 \\
& & \pm 0.023 & \pm 0.039 & \pm 0.019 & \\
NGC 7192 & -0.049 & +0.004 & $\ldots$ & -0.035 & 74 \\
& \pm 0.033 & \pm 0.025 & & \pm 0.022 & \\
\hline
\end{tabular}

near-infrared-optical colours of globular clusters in NGC 3115 , NGC 5846, NGC 7192 we refer the reader to Puzia et al. (2002a) and Hempel et al. (2003).

Most of the colour distributions are consistent with previous colour-distribution studies (Gebhardt \& Kissler-Patig 1999; Larsen et al. 2001; Kundu \& Whitmore 2001a,b), but only where the latter had large enough sample size. Our photometry goes deep (reaching the GCLF turn-over in most cases, see Fig. 6) and our field sampling $\left(6.7^{\prime} \times 6.7^{\prime}\right)$ is large enough to cover a representative fraction $\left(\geq 51 \%^{1}\right)$ of the observed globular cluster system. For instance, the $V-I$ colour distribution of NGC 3379 gained a substantial amount of blue globular clusters $(\sim 15 \%$ of the entire population down to $V=23.5)$ which have not been included in previous HST/WFPC2 studies (e.g. Larsen et al. 2001). This is likely to be due to a significant difference in spatial distribution of red and blue globular clusters in this galaxy. There is evidence that red clusters are more concentrated towards the center than the blue globular cluster sub-population which rather resides in the halo (see Sect. 2.4). This illustrates that colour distributions which were created from photometric data of limited field size (e.g. HST/WFPC2) might be misleading if significant differences in spatial distributions of globular cluster sub-populations are present.

\subsection{Optical/near-infrared colour-colour diagrams}

We combine now our optical FORS2 photometric data with recently published near-infrared data for NGC 3115 (Puzia et al. 2002a), NGC 5846, and NGC 7192 (Hempel et al. 2003) and construct optical/near-infrared two-colour diagrams of candidate and confirmed globular clusters. All near-infrared data were obtained with the ISAAC instrument attached to ESO's VLT with a $2.5^{\prime} \times 2.5^{\prime}$ field of view. Figure 2 shows $I-K$ vs. $B-K$ diagrams with spectroscopically confirmed globular

\footnotetext{
1 The fraction was determined with the surface density profiles found in Sect. 2.4.
}

clusters marked as asterisks. Due to the smaller field of view of the near-IR data, these two-colour diagrams are restricted to the central regions of each galaxy and do not cover a representative of the entire globular cluster system (see discussion in Sect. 2.2). Although optical/near-infrared colours are powerful metallicity discriminators (e.g. Puzia et al. 2002b), they are of limited use for the slit mask design due to the very constrained field of view.

However, a posteriori it is worthwhile to compare optical/near-infrared colours of globular clusters, background galaxies and foreground stars in order to minimise contamination of the candidate selection. Based on our set of photometric passbands $(B, V, R, I, K)$, we find that the combination of $I-K$ and $B-K$ separates globular clusters from stars and galaxies most reliably. In the following we describe how to reduce the contamination of globular cluster candidate samples by foreground stars and background galaxies.

\subsubsection{Eliminating foreground stars}

Globular clusters fall on a rather narrow sequence in the $I-K$ vs. $B-K$ diagram (hatched region in Fig. 3 ). In general, at similar $I-K$ colours, cool giant stars lack $B$ band flux compared to the integrated light of globular clusters and have therefore redder $B-K$ colours. As the effective temperature of red giants is a sensitive function of metallicity, more metal-poor (warmer) stars will be more likely to resemble mean globular cluster colours. This is shown in the left panel of Fig. 3. It is important to keep in mind that age and metallicity of contaminating stars depend on the sampled galactic coordinates. In order to illustrate the difference in colour between disk and halo stars in the $I-K$ vs. $B-K$ diagram we plot colours of stars found in the FORS Deep Field (FDF, galactic coordinates $l=191.40^{\circ}$, $b=-86.46^{\circ}$; Heidt et al. 2003; Gabasch et al. 2004) and representative colours of disk dwarf, giant, and supergiant stars in the solar neighborhood (Cox 2000). While the former sample is likely to be dominated by old metal-poor halo stars ${ }^{2}$, the latter data resemble colours of metal-rich disk stars. To show the metallicity offset between metal-poor and metal-rich stars more clearly we plot all stars found in the FDF field with a field-of view of $\sim 39$ square arcminutes (indicated by stars in Fig. 3). Thus, number counts have to be rescaled as the field of view of our combined optical/near-infrared photometry is only 6.25 square arcminutes. Metal-rich disk stars can be reliably separated from globular cluster candidates as the former are significantly redder in $B-K$. The colours of metal-poor halo stars, on the other hand, are more similar to globular cluster colours. However, the surface density of these stars with metallicities $[\mathrm{Fe} / \mathrm{H}] \lesssim-1.5$ is relatively low, of the order of one star per field-of-view in our optical data ( $\sim 5$ square arcminutes) in the range $18 \leq V \leq 22.5$, with little dependence on galactic coordinates (Robin et al. 1996).

In general, the combination of optical and near-infrared photometry provides a good discriminator to distinguish between globular clusters and metal-rich foreground stars. However, the colours of metal-poor globular clusters can be

2 The FDF line of sight is almost perpendicular to the Galactic disk. 

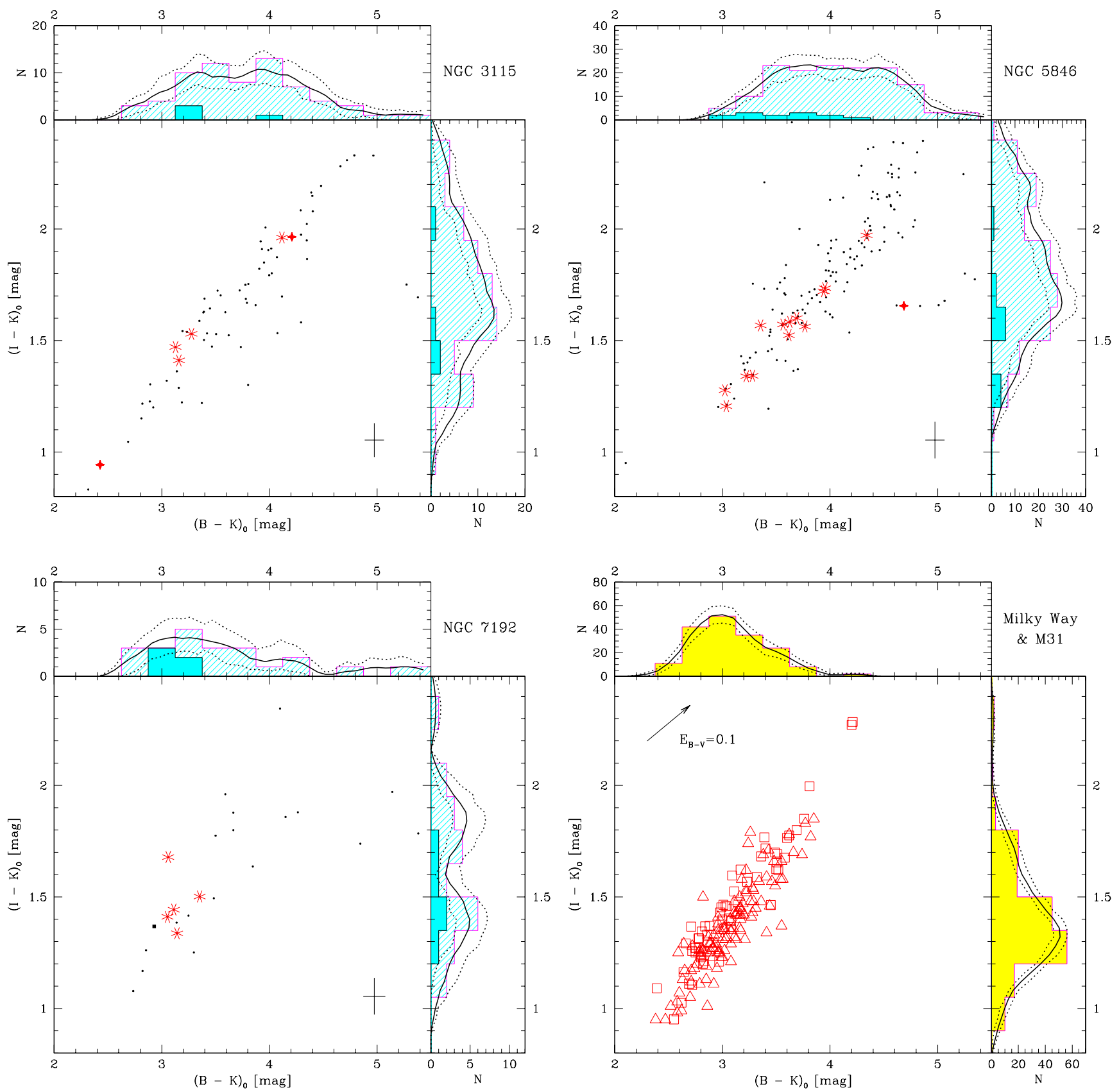

Fig. 2. Optical/near-infrared $I-K$ vs. $B-K$ two-colour diagrams for objects in NGC 3115, NGC 5846, and NGC 7192. Near-infrared data for NGC 3115 were taken from Puzia et al. (2002a) while the near-infrared data for NGC 5846 and NGC 7192 were taken from Hempel et al. (2003). Average photometric errors are indicated in the lower right corner of each diagram. Asterisks indicate spectroscopically confirmed globular clusters. 4-prong stars and solid squares show colours of confirmed foreground stars and background galaxies, respectively. Hatched and solid histograms in the sub-panels show the colour distributions of all objects and of spectroscopically confirmed globular clusters. Solid lines within the sub-panels are probability density estimates with their $90 \%$ confidence limits (dotted lines). In the lower right panel we show colours of globular clusters in M 31 (triangles) and the Milky Way (squares). Optical colours for Milky Way globular clusters were taken from Harris (1996), near-infrared colours were adopted from Aaronson \& Malkan (in preparation). The data for M 31 globular clusters are from Barmby et al. (2000).

mimicked by metal-poor halo stars. These stars need to be sorted out by other selection criteria such as magnitude. Based on the FDF data and the Galactic stellar population model (Robin et al. 1996), we expect a total stellar contamination of $\lesssim 1-10 \%$ (depending on the richness of the globular cluster system) at galactic latitudes $|b| \gtrsim 40^{\circ}$. At lower latitudes the foreground contamination is rising.

\subsubsection{Eliminating background galaxies}

Another source of contamination are background galaxies. To estimate their $I-K$ and $B-K$ colours, we use a subsample of the FDF data which corresponds to our combined optical/near-infrared data in field-of-view size and photometric completeness (mainly limited by the near-infrared photometry 

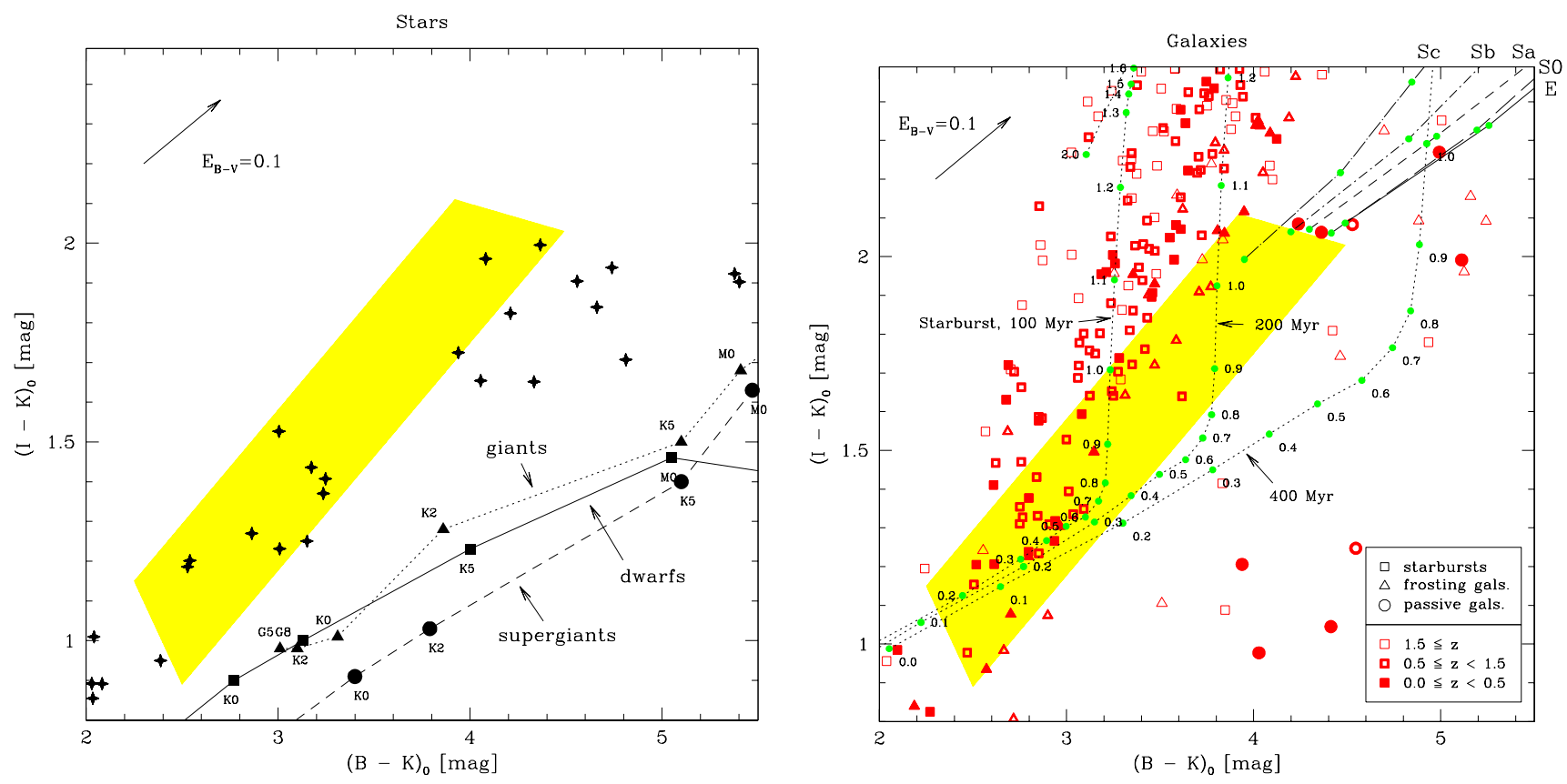

Fig. 3. This figure compares in the two-colour diagram the position of globular clusters (indicated by a shaded region, see also Fig. 2) versus the mean locus of possible contaminating objects, such as foreground stars (left panel) and background galaxies (right panel). Left panel: Measured colours of stars were taken from the FORS Deep Field (Heidt et al. 2003; Gabasch et al. 2004) and are indicated by 4-prong stars. Due to their small surface density, we use the full set of stars found in the FDF which has a field-of view of $\sim 39$ square arcminutes. Number counts have to be corrected when contamination estimates are performed for our sample (field of view 6.25 square arcminutes for the $K$-selected data, $\sim 45$ square arcminutes for the optical data). Spectral sequences of dwarf, giant, and supergiant stars in the disk are shown as solid, dotted, and dashed lines, respectively, and were taken from Cox (2000). Spectral types are indicated along each sequence. The offset between FDF stars and the spectral sequences is due to a higher metallicity of the latter which were constructed from stars in the solar neighbourhood. Right panel: Open squares mark starbursts, open triangles are galaxies in which star-formation ceased a few Gyrs ago (so-called "frosting" galaxies), while open circles show colours of old, passively evolving elliptical galaxies. The full data set from the FDF was used $\left(I_{50 \% \text { compl. }} \approx 26.37\right.$, Heidt et al. 2003) and thinned so that expected number counts in the field-of-view of the $K$-selected data can be directly read off. The photometrically determined redshift of galaxies is indicated by the filling factor of symbols (see legend in the lower right corner). Additionally, we k-correct the colours for E, S0, Sa, Sb, and Sc galaxies using template spectra of Mannucci et al. (2001) and Kinney et al. (1996). The sequences are labeled in the upper right corner of the right panel. Shaded dots along the sequences indicate redshifts which increase from $z=0.0$ in steps of $\Delta z=0.1$ towards redder colours. Dotted lines show the evolution of k-corrected colours for starburst galaxies with an age of 100, 200, and 400 Myr using templates from Maraston (2003, in preparation). Redshifts are marked along each sequence.

at $K \sim 21.5)$. In the right panel of Fig. 3 we plot $I-K$ vs. $B-K$ colours of galaxies with high star-formation rate (open squares), galaxies in which star formation ceased a few Gyrs ago (so-called "frosting" galaxies, open triangles), and passively evolving ellipticals (open circles) (see Heidt et al. 2003; Gabasch et al. 2004, for a quantitative classification). Judging from the FDF data in Fig. 3, we find that the blue part of the mean globular clusters locus, indicated by the shaded region, is mainly contaminated by starburst galaxies while the red part is prone to contain "frosting" galaxies. Depending on the boundary definitions, we find $\sim 20-30$ starbursts and $\sim 10-15$ "frosting" galaxies inside the region where globular clusters are preferentially found. However, most of these galaxies would be resolved in our photometry (typical seeing $\leq 1^{\prime \prime}$ ) and rejected by the FWHM (or size) selection.

Potentially problematic objects are distant starburst galaxies which are barely resolved and still bright enough to be classified as globular cluster candidates. At redshift unity, one arcsecond corresponds to $\sim 8 \mathrm{kpc}$ in a flat $\Lambda$-universe with $\Omega_{m}=0.3$ and $H_{0}=70 \mathrm{~km} \mathrm{~s}^{-1} \mathrm{Mpc}^{-1}$ (at $z=2$, one arcsecond covers $\sim 8.4 \mathrm{kpc}$ ). Typical sizes of distant starburst galaxies range between a few hundred pc to a few kpc (e.g. Guzman et al. 1998; Soifer et al. 2001) and can be reliably resolved with ground-based photometry up to $z \approx 0.1$. At $z \approx 1.0$ even the brightest starbursts with typical absolute magnitudes $M_{V} \approx$ -21 up to -22 mag are already too faint to enter our magnitude selection (cut at $V=23 \mathrm{mag}$ ). We conclude that provided good photometric quality (seeing $\leq 1^{\prime \prime}$ ), starbursts are reliably rejected by the combination of $F W H M$ and magnitude selection below $z \approx 0.1$ and above $z \approx 1.0$. Optical/near-infrared colours can be a good additional discriminator for the remaining intermediate-redshift space as shown in the following.

We simulate the redshift evolution (both k-correction and luminosity corrections) of optical/near-infrared colours of a $100,200,400$, and 800 Myr old starburst using template spectra taken from Maraston (2003, in preparation). The latter templates include the stellar evolutionary phase of thermally pulsing AGB stars, that dominates the infrared and bolometric flux at these ages (e.g. Maraston et al. 2001). We use spectra of instantaneous star-formation with a metallicity $[\mathrm{Z} / \mathrm{H}]=-0.33$. 
We find that young starbursts between 100 and 200 Myr produce colours which are consistent with globular cluster colours in the redshift range between $z=0.1$ and $z=1.0$. As most starbursts are intrinsically located in high-reddening regions, we note that bright unresolved starbursts with ages $\leq 100 \mathrm{Myr}$ and reddening values $E_{B-V}>0.1$ can show typical globular cluster colours, as well. Those objects could contaminate the blue sub-sample of globular cluster candidates (see reddening vector in the right panel of Fig. 3). Starburst older than $300 \mathrm{Myr}$ have colours that are inconsistent with globular cluster colours beyond a redshift $z \approx 0.2$. Indeed, at these ages the AGB-phase transition boosts both colours to $I-K \gtrsim 2.5$ and $B-K \gtrsim 4.0$.

The k-corrected colours for galaxies are simulated with empirical template spectra of Mannucci et al. (2001) and Kinney et al. (1996) for elliptical, lenticular, and spiral galaxies. To account for the luminosity evolution requires to adopt a model for these galaxies and goes far beyond the aim of this exercise. The k-correction paths are shown in the right panel of Fig. 3 with redshifts indicated by filled dots starting at $z=0.0$ and increasing in steps of $\Delta z=0.1$ to redder colours. It is obvious that non-starforming early-type galaxies entirely avoid the mean colour locus of globular clusters at all redshifts. Colours of low- $z$ later-type galaxies are only marginally consistent with the reddest globular clusters. Sc galaxies below $z=0.1$ intersect the shaded region where red globular clusters are found. However, these galaxies are efficiently rejected by the FWHM selection.

We conclude that the $I-K$ vs. $B-K$ diagram allows one to reliably disentangle globular cluster candidates from foreground disk stars as well as early-type and spiral galaxies with no or little on-going star-formation. Remaining potential contaminants are unresolved starbursts with ages $\$ 300 \mathrm{Myr}$ at intermediate redshifts between $z \approx 0.1$ and $\sim 1.0$. Based on FDF data we expect $\sim 35-45$ background galaxies down to $I=22.5$ within the FORS field-of-view ( $\sim 45$ square arcminutes) with colours resembling those of globular clusters. The majority of these galaxies is resolved and rejected by the PSF selection. Indeed, the only background galaxy found in our $K$-selected sample with optical/near-infrared colours (4\% of the sample; a fill-in object with $V=22.83, B-K=3.06$, and $I-K=1.42$ ) is consistent with a young unresolved starburst galaxy at $z \approx 0.14$. The spectrum has too low $S / N$ to allow a more accurate classification based on spectral features. The corresponding absolute magnitude of the object is $M_{V} \approx-16.3$ and is consistent with a SMC-type galaxy $\left(M_{V}=-16.2 \mathrm{mag}\right.$ Binney \& Merrifield 1998).

The overall surface density of contaminating objects with globular-cluster colours and magnitudes which are bright enough for integrated-light spectroscopy $(V \gtrsim 22-23$ at 8-10 m-class telescopes with colours as indicated in Fig. 3) is negligible ( $\$ 10 \%$ ) where the surface density of globular clusters is high, that is within $\sim 1 R_{\text {eff }}$. Multi-object spectroscopic studies are therefore expected to have a highest success rates in central regions of a globular cluster system. We refer to Sect. 4.2 for an analysis of the success rate of the candidate selection.

\subsection{Radial surface density profiles of globular cluster systems}

In future papers we will study the properties of globular cluster systems as a function of their host's properties. To derive host galaxy masses we use globular clusters as tracer particles of the galaxy potential in Sect. 4.3. The tracer mass estimator requires the knowledge of the surface-density profiles of the tracer-particle population. Our photometric data probe a large enough range in galactocentric radius to reliably sample the globular cluster surface density profile. Moreover, in some globular cluster system formation scenarios the surface density profile of blue and red clusters is expected to change differently as a function of radius (e.g. Ashman \& Zepf 1992; Côté et al. 1998). It is therefore important to study the profiles of each globular cluster sub-sample to control radial biases of further analyses.

In order to derive surface-density profiles we use the IRAF task ELLIPSE and the pre-selected FORS2 photometric sample (see Sect. 2.2). First, we model the surface-brightness profiles of the galaxy light on images which feature the best seeing and which were cleaned of point sources. The same elliptical isophotes are used for surface-brightness profiles in other passbands and to construct the surface-density profile of globular-cluster candidates. Colour-magnitude diagrams in Fig. 1 show that photometric completeness as a function of colour does not greatly affect the radial surface-density profiles as the change in completeness level is negligible within the colour region from which globular clusters are selected.

In Fig. 4 we plot surface density profiles of globular cluster candidates. Prior to creating the profiles, we excluded extremely blue objects (which are likely contaminating fore/background sources, see Sect. 2.3) by applying cuts at $V-I=$ 0.3 (NGC 3379), $V-I=0.7$ (NGC 2434, NGC 7192), $V-I=0.8(\mathrm{NGC} 1380, \mathrm{NGC} 3115$, NGC 5846), and $B-I=1.3$ (NGC 3585). Objects with colours redder than $V-I=1.3$ (NGC 2434, NGC 3379), $V-I=1.4$ (NGC 1380, NGC 3115), $V-I=1.5(\mathrm{NGC} 5846), V-I=1.6(\mathrm{NGC} 7192)$, and $B-I=2.3$ (NGC 3585) were also rejected. Outermost isophotes were used to subtract the background light and the surface density of background objects. Depending on the distance of the galaxy, the outermost accessible radii vary between $\sim 10$ and $\sim 50 \mathrm{kpc}$, which corresponds to $R \geq 2 R_{\text {eff }}$ for all sample galaxies. Henceforth we compare only the slopes of the surface brightness profiles of the galaxy and the surface density profiles of globular cluster systems. Within the range of our globular cluster data colour-index changes of the diffuse light are negligible and we representatively use the $I$ band surface brightness profile, since $I$-band data is available for all our sample galaxies. In order to have a fair comparison of the slopes we plot $\log \left(N_{\mathrm{GC}} / \operatorname{arcsec}^{2}\right)$ and $\mu_{I} / 2.5$ versus the logarithmic semi-major axis galactocentric distance (Fig. 4). However, this procedure compares globular cluster number counts with a luminosity density of the host galaxy and is only valid if the mean $\mathrm{M} / \mathrm{L}$ ratio of globular clusters remains constant as a function of radius. The mean $\mathrm{M} / \mathrm{L}$ ratio is subject to change when young globular clusters are concentrated at a given radius. However, we expect that the majority of the globular cluster system is old 

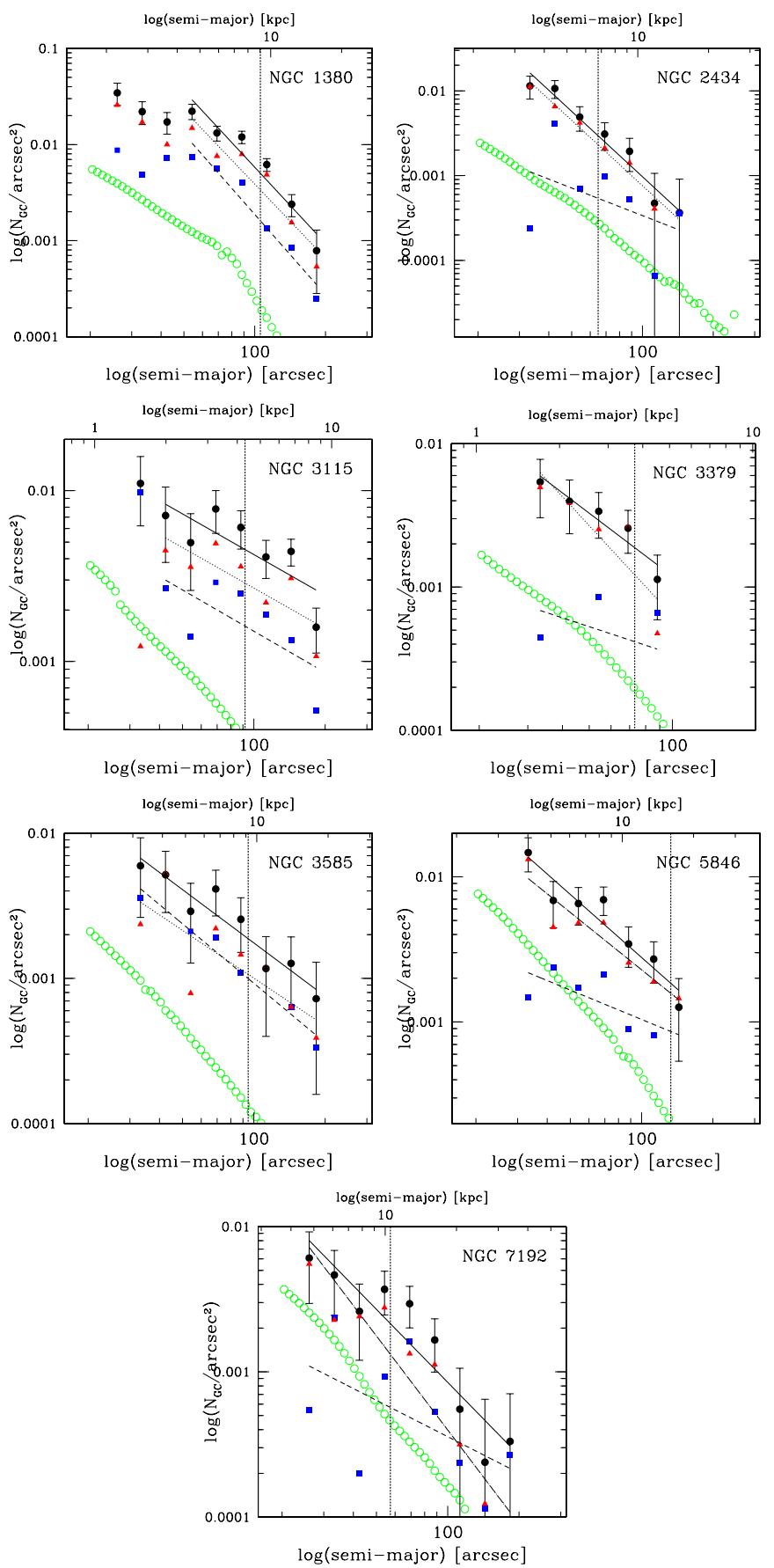

Fig. 4. Surface density and surface brightness profiles of globular cluster systems and their host galaxies. The surface density of all globular cluster candidates is indicated by black dots with a power-law fit indicated as a solid line. Multi-modal colour distributions were divided at $V-I=1.1$ or $B-I=1.8$ into blue and red globular clusters. Powerlaw fits to the blue and red sub-populations are shown as dashed and dotted lines, respectively. Open circles show the scaled surface brightness profile of the host galaxy derived from $I$ band photometry. Both profiles were calculated using the same isophotes. A vertical line indicates the effective radius of each system taken from de Vaucouleurs et al. (1991). Poisson errors are indicated for the total surface density profiles.

and that the comparison of the slopes is acceptable to the first order.
Table 4. Power-law exponents, $\Gamma$, of globular-cluster surface density profiles, $\Sigma(R) \sim R^{-\Gamma}$, of globular cluster systems and sub-populations. The second and third column shows the slopes for the profiles of the blue and red sub-populations, respectively. In the same order, these slopes are indicated as dashed and dot-dashed lines in Fig. 4. The last column are the slopes of the entire cluster system. All given errors are uncertainties of the fit. Numbers in parentheses indicate a brute-force division of globular cluster systems which show a single-peak colour distribution in a blue and red globular cluster sub-population (see text for details).

\begin{tabular}{lccc}
\hline \hline Galaxy & Blue & Red & All \\
\hline NGC 1380 & $2.78 \pm 0.33$ & $2.56 \pm 0.42$ & $2.63 \pm 0.37$ \\
NGC 2434 & $(1.08 \pm 0.98)$ & $(2.56 \pm 0.24)$ & $2.56 \pm 0.25$ \\
NGC 3115 & $0.80 \pm 0.36$ & $0.79 \pm 0.26$ & $0.79 \pm 0.28$ \\
NGC 3379 & $(0.64 \pm 0.84)$ & $(2.09 \pm 0.67)$ & $1.46 \pm 0.28$ \\
NGC 3585 & $1.36 \pm 0.12$ & $1.09 \pm 0.35$ & $1.22 \pm 0.20$ \\
NGC 5846 & $(0.67 \pm 0.35)$ & $(1.31 \pm 0.21)$ & $1.44 \pm 0.33$ \\
NGC 7192 & $(0.82 \pm 0.48)$ & $(2.15 \pm 0.28)$ & $1.67 \pm 0.24$ \\
\hline
\end{tabular}

The surface-density profile of each globular-cluster system appears generally comparable to or less steep than the galaxy light profile. In the case of the two S0 galaxies, NGC 1380 and NGC 3115, both globular-cluster profiles might be influenced towards the center by the presence of a stellar disk (Bothun \& Gregg 1990). Disk shocking and disk instabilities might be responsible for enhanced cluster destruction and could decrease the globular cluster surface density close to the center (Gnedin $\&$ Ostriker 1997). Another consequence of the disk is a reduced photometric completeness in the central parts of the galaxy. We exclude central regions inside $\sim 0.5 R_{\text {eff }}$ from the profile fitting for these two galaxies. In fact, the surface-density profile of globular clusters in NGC 1380 tends to be less steep inside $R_{\text {eff }}$ compared to radii larger than one effective radius. Globular cluster systems in elliptical galaxies are less affected by dynamical erosion or by a varying photometric completeness.

We fit power-law profiles of the form $\Sigma(R) \sim R^{-\Gamma}$ to the selected globular cluster data (solid circles in Fig. 4). Where colour multi-modality is apparent (see Sect. 2.2), we divide each candidate sample into a blue (solid squares) and red (solid triangles) globular clusters with a cut at $V-I=1.1$ (NGC 1380, NGC 3115) and $B-I=1.8$ (NGC 3585) and fit power-law profiles to each sub-sample and to the whole globular cluster system. Profiles for blue sub-samples are plotted as dashed lines. Dotted lines indicate profiles for red globular cluster candidates. Table 4 summarises the slopes for each galaxy.

The power-law exponents of the globular cluster surfacedensity profiles cover a wide range from $\Gamma \approx 0.8$ to steep profiles with $\Gamma \approx 2.6$. Blue and red globular cluster subpopulations appear to have similar profiles in all multi-modal galaxies. Although less significant, different power-law exponents are found for blue and red globular clusters (cuts at $V-I=0.95$ for NGC 2434, $V-I=0.8$ for NGC 3379, and $V-I=1.1$ for NGC 5846 and NGC 7192) in galaxies with a single-peak colour distribution. In all cases the metal-poor globular-cluster system is more spatially extended than its metal-rich counterpart. The absolute cluster number 
densities of blue and red clusters reach comparable values at large radii $\gtrsim 1 R_{\text {eff }}$. This inevitably leads to the fact that in unimodal galaxies red globular clusters dominate our spectroscopic samples close to the center, while blue clusters are preferentially selected in the halo at large radii. This very interesting result requires a more detailed analysis and must be considered when radial analyses of globular cluster systems are performed.

\subsection{Selection of globular cluster candidates for spectroscopy}

For the selection of globular cluster candidates for spectroscopic follow-up we focus on objects with colours representative of high-density regions of a given colour distribution (see Fig. 1). Compliant with the restrictions of the slit-mask design (non-uniform spatial coverage of galactocentric radii, minimum slit length for good sky subtraction, limited deviation from the mask meridian for sufficient wavelength coverage, etc.) we representatively sample the underlying colour distributions of each globular cluster system. Furthermore, we focused primarily on objects inside one effective radius where the surface density of clusters is relatively high compared with surface densities of foreground stars and background galaxies.

Another constraint results from the faint magnitudes of the cluster candidates. To increase the likelihood of selecting a globular cluster we assigned a high priority to objects with magnitudes around the expected turn-over of the globular cluster luminosity function (GCLF). This however, has to be traded-off with the minimum $S / N$ of $\sim 20 \AA^{-1}$ which is required for reliable index measurements. Overall the limiting magnitude was adjusted to $V=23 \mathrm{mag}$ and was exceeded only in a few cases where the slit-mask design forced it.

In particular, our first-choice targets were drawn from the pre-selected sample (see Sect. 2.2) in the colour range $0.8 \lesssim$ $V-I \lesssim 1.3$, and where colour information was available, from $1.5 \lesssim B-I \lesssim 2.5$ and $1.0 \lesssim B-R \lesssim 1.7$. According to simple stellar-population models of Maraston et al. (2003) these colour ranges are expected for stellar populations with metallicities $[\mathrm{Z} / \mathrm{H}] \gtrsim-1.0$ between $\sim 1$ and $\sim 15$ Gyr. These cuts exclude clusters with very low metallicities $(\lessgtr-1.5 \mathrm{dex})$ which have ages less than $\sim 5$ Gyr. Before the final selection, all colours of cluster candidates were corrected for the respective foreground extinction taken from Schlegel et al. $(1998)^{3}$.

In general, the upper selection criteria favour globular clusters which are brighter than the GCLF turn-over. If young ( $\$ 5 \mathrm{Gyr}$ ) globular clusters are present, they will be preferentially selected compared to old globular clusters due to their brighter magnitudes. In that case, our sample is likely to be biased towards young metal-rich clusters at ages $\lesssim 5 \mathrm{Gyr}$.

First-choice candidates are used to create the slit masks. Remaining gaps in-between two slits are filled by objects which suffice slightly relaxed selection criteria. To fill the slit masks

\footnotetext{
${ }^{3}$ Even for the highest-z galaxy in our sample, NGC 7192, the $\mathrm{k}$-corrections of colours used for candidate selection are of the order of a few hundredths mag. Hence, we do not consider these negligible colour corrections.
}

most efficiently, we relaxed the magnitude limit and the $F W H M$ cut to include also faint objects. More than $50 \%$ of these fill-in objects was found to be genuine globular clusters.

\section{Spectroscopic data}

We created two slit masks for NGC 1380 and NGC 2434 with 98 and 100 objects in total, respectively. For NGC 3115 we used the MOS unit of FORS2 with the 19 slits aligned to cover 22 objects. For NGC 3379, NGC 3585, NGC 5846, and NGC 7192 we designed one slit mask each to take spectra of $34,35,39$, and 34 globular cluster candidates, respectively. In total we obtained spectra for 362 globular cluster candidates in seven early-type galaxies.

All data were obtained with the FORS2 instrument at UT2 (unit telescope 2, Kueyen) of ESO's VLT. The data of period 65 (NGC 3115) were taken with the multi-object slit (MOS) unit with 19 movable slits. In period 66 and 67 spectroscopic observations were carried out with the mask-exchange unit (MXU). A MOS mask with its 19 movable slits restricts the observations to a limited amount of objects per exposure. The MXU unit, instead, increases the multiplexity by at least a factor of two allowing for simultaneous spectroscopy of up to $\sim 40 \mathrm{ob}-$ jects per frame. The total exposure time for each individual mask was adjusted according to the observing conditions and the magnitudes of selected objects. Typical exposure times vary between $\sim 3$ and $\sim 9$ hours per mask. The observations were split into sub-integrations of $1800 \mathrm{~s}$ (see Table 5 for details). All exposures used the $600 \mathrm{~B}+22$ grism with 600 grooves per mm resulting in a dispersion of $1.2 \AA$ per pixel $(R \sim 780)$ on a $2048 \times 2048$ pix $^{2}$ thinned Tektronix CCD chip with $24 \mu \mathrm{m}$ pixels. The readout was done in a single-channel mode. In period 65 this resulted in $5.2 \mathrm{e}^{-}$readout noise with a gain $1.85 \mathrm{e}^{-} /$ADU. Observations in period 66 and 67 had $5.41 \mathrm{e}^{-}$ readout noise and a $1.91 \mathrm{e}^{-} / \mathrm{ADU}$ gain. All observations use a slit width of $1^{\prime \prime}$. The mean wavelength coverage of the system is $\sim 3450-5900 \AA$ with a final resolution of $\sim 5 \AA$.

\subsection{Spectroscopic data reduction}

All spectra were processed with standard reduction techniques implemented in IRAF ${ }^{4}$. In summary, after subtracting a masterbias all frames were divided by a normalized flat-field image. The residual gradients on the normalized flat were found to be smaller than $0.5 \%$. The resulting images were cleaned off cosmics with the routine by Gössl \& Riffeser (2002) employing a 9- $\sigma$ threshold and a characteristic cosmic-ray FWHM of 1.1 pixel. Optical field distortions in the FORS field-of-view bend spectra which lie away from the optical axis. This effect complicates an accurate sky subtraction as tilted sky lines would be incorrectly subtracted when a central wavelength solution is applied to the object's slit aperture. We have, therefore, calculated a wavelength solution for each pixel row from arclamp spectra and rectified all slit spectra according to this

\footnotetext{
4 IRAF is distributed by the National Optical Astronomy Observatories, which are operated by the Association of Universities for Research in Astronomy, Inc., under cooperative agreement with the National Science Foundation.
} 
Table 5. Journal of spectroscopic observations. Exposure times are given in seconds. Two masks were used for NGC 1380 and NGC 2434 . All slit-mask observations were performed with the FORS mask exchange unit (MXU), except for NGC 3115 where we used 19 movable slits of the FORS instrument to create a slit mask (MOS mode). Note that no longslit spectroscopy (LSS) was obtained for NGC 3115.

\begin{tabular}{|c|c|c|c|c|}
\hline Galaxy & Program no. & Nights & MOS/MXU exp. time & LSS exp. time \\
\hline NGC 1380 & P66.B-0068 & 28th-31st Dec. 2000 & $\begin{array}{l}\text { MXU mask1: } 8 \times 1800 \\
\text { MXU mask2: } 6 \times 1800\end{array}$ & $4 \times 1800$ \\
\hline NGC 2434 & P66.B-0068 & 28th-31st Dec. 2000 & $\begin{array}{l}\text { MXU mask1: } 8 \times 1800 \\
\text { MXU mask2: } 6 \times 1800\end{array}$ & $4 \times 1800$ \\
\hline NGC 3115 & P65.N-0281 & 5th \& 6th May 2000 & MOS mask: $6 \times 1800$ & $\ldots$ \\
\hline NGC 3379 & P66.B-0068 & 28th-31st Dec. 2000 & MXU mask: $8 \times 1800+1200$ & $3 \times 1800$ \\
\hline NGC 3585 & P67.B-0034 & 26th-29th May 2001 & MXU mask: $15 \times 1800$ & $5 \times 1800$ \\
\hline NGC 5846 & P67.B-0034 & 26th-29th May 2001 & MXU mask: $18 \times 1800$ & $5 \times 1800$ \\
\hline NGC 7192 & P67.B-0034 & 27th-29th May 2001 & MXU mask: $17 \times 1800+900$ & $6 \times 1800+900$ \\
\hline
\end{tabular}

distortion mask. Subsequently, the IRAF task APALL was applied to the rectified MOS/MXU images and used to define object and background apertures. The upper and lower boundaries of an object aperture were adjusted so that the object flux was still higher than the adjacent sky noise level. Limits at $\sim 15 \%$ of the peak flux were found to be optimal for all aperture boundaries. The same task was also used to trace the apertures along the dispersion axis and optimally extract the object flux according to Horne (1986). During the extraction procedure the sky is modeled in one dimension perpendicular to the dispersion axis by a linear relation with a $\kappa-\sigma$ clipping to remove residual bad pixels. Finally, all spectra are transformed into wavelength space with an accuracy better than $0.1 \AA$ using a low-order spline. Subsequently, we used the flux standards EG21, Feige56, Feige110, LTT377, LTT1020, LTT1788, and LTT3864 to transform raw counts into flux units.

From the different exposures we average all single spectra of each object. Due to varying observing conditions (seeing, atmospheric transparency, alignment of slit masks, etc.), the spectra of each sub-integration series have changing signal-tonoise ratios $(S / N)$. To obtain a final spectrum with the highest possible $S / N$, we average all single spectra of each given object and weight them by their individual $S / N$. To determine the weights we calculate the $S / N$ for each spectrum in the range around $5000 \AA$. The change in $S / N$ between the final spectra with and without weighting is $\sim 10 \%$. The following analysis steps make use of the optimally combined spectra.

\section{Kinematics}

\subsection{Radial velocities}

Radial velocities $\left(v_{\mathrm{r}}\right)$ are measured by cross-correlating the combined candidate spectra with high- $S / N$ spectra of two M31 globular clusters, 158-213 and 225-280 (Huchra et al. 1982) using the IRAF task FXCOR. The resulting heliocentric radial-velocity histograms are shown in Fig. 5. The plotted distributions are clearly concentrated around the mean systemic radial velocity measured from the diffuse light (de Vaucouleurs et al. 1991). We define bona-fide globular clusters with radial velocities which are off by maximally $\pm 400 \mathrm{~km} \mathrm{~s}^{-1}$ of the mean systemic $v_{\mathrm{r}}$. For most galaxies the distinction between globular clusters and contaminants such as foreground stars and background galaxies is unequivocal since the latter have either much lower or higher $v_{\mathrm{r}}$. Exceptions are two objects in NGC 3115.

In the case of NGC 3115 , objects \#10 and \#15 have relatively low radial velocities $v_{\text {rad, } 10}=344 \pm 48$ and $v_{\text {rad, } 15}=285 \pm$ $19 \mathrm{~km} \mathrm{~s}^{-1}$, respectively. Assuming a simple rotation model of the Milky Way the mean streaming velocity of foreground stars in the direction of NGC $3115\left(l=247.78^{\circ}, b=36.78^{\circ}\right)$ is $v_{\text {rad }}=220 \cdot \sin (2 l) \cos ^{2}(b) \approx 100 \mathrm{~km} \mathrm{~s}^{-1}$ (van de Kamp 1967). It is therefore unlikely that the two objects are foreground disk stars. Both are, however, not completely inconsistent with highvelocity stars in the galactic halo. Within the colour limits of our selection, 32 stars with magnitudes between $V \approx 21.5$ and $\sim 22.5$ are expected ${ }^{5}$ in the direction of NGC 3115 within the FORS2 field of view (Robin et al. 1996). To decide more conclusively whether the two objects are foreground stars or genuine globular clusters their spectra deserved a detailed investigation. However, the result was inconclusive (see Appendix A).

We conclude that the two objects cannot be assigned confidently to either of the two groups; stars or globular clusters. Both spectra are, therefore, kept in the globular cluster sample but we flag them as problematic.

Using radial velocities we confirm 43 globular clusters in NGC 1380, 6 in NGC 2434, 18 in NGC 3115, 18 in NGC 3379, 20 in NGC 3585, 28 in NGC 5846, and 10 globular clusters in NGC 7192.

\subsection{Success rates of globular-cluster candidate selection}

In the following, we calculate the success rate of our candidate selection. For each galaxy, the success rate varies with changing surface density of globular clusters and therefore with galactocentric radius. It is instructive to calculate the success rate for our entire sample and inside one effective radius $R_{\text {eff }}$, with and without our colour pre-selection. In other words, with and without fill-in objects. All values are summarised in Table 6. In general the success rates drop for larger galactocentric radii.

\footnotetext{
5 Model predictions were calculated using the code available at www. obs-besancon. fr/www/modele/modele_ang.html
} 

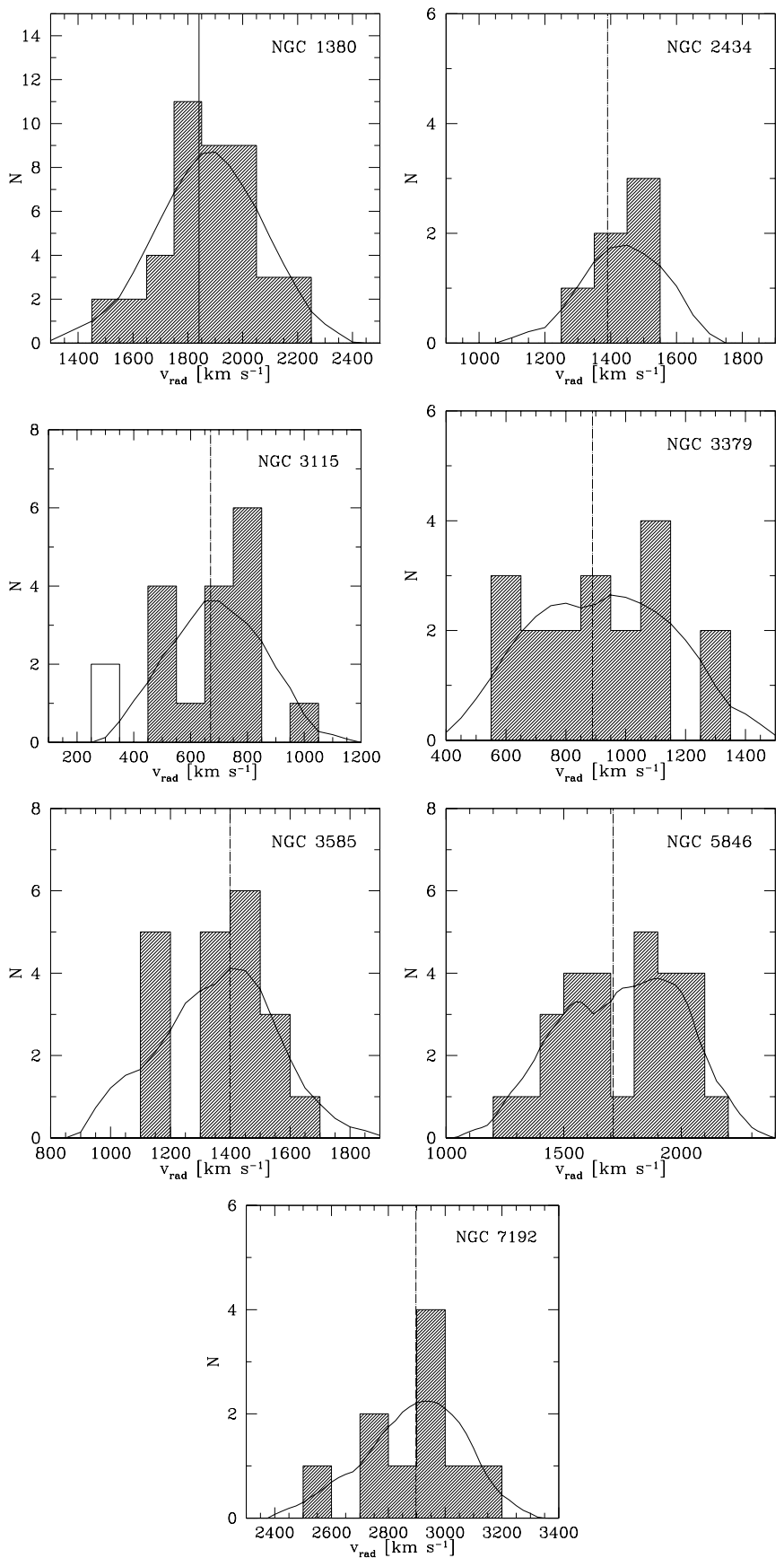

Fig. 5. Radial velocity histograms for globular clusters in all studied galaxies. The mean heliocentric radial velocity of the host was determined in the optical and is shown as a vertical dot-dashed line taken from the RC3 catalog (de Vaucouleurs et al. 1991). The solid line is a probability density estimate using an Epanechnikov kernel with a fixed width $\delta v_{\text {rad }}=100 \mathrm{~km} \mathrm{~s}^{-1}$ (for details see Silverman 1986). Two ambiguous objects in NGC 3115 are indicated by an open histogram (see also Sect. 4.1).

The major fraction of contaminants are foreground stars, thus it is not surprising that the success rate correlates with galactic latitude. High success rates $(\gtrsim 80 \%)$ are guaranteed if colour selection is applied and the field of view of the spectrograph covers about one to two effective radii, provided that the host galaxy is located at high galactic latitudes $\left(|b| \gtrsim 40^{\circ}\right)$. Only NGC 2434 suffers from severe foreground contamination.
Table 6. Success rates of photometric globular cluster selection as a function of galactocentric radius for the entire sample and inside one effective radius. The numbers give the fraction of confirmed globular clusters with respect to the total number of objects for which spectroscopy was obtained without and including colour selection of candidates (marked by the index "sel"). The effective radii were taken from the RC3 catalog. Success rates for NGC 3115 were calculated including object \#10 and \#15.

\begin{tabular}{lcccc}
\hline \hline Galaxy & Total & $<1 R_{\text {eff }}$ & Total $_{\text {sel }}$ & $<1 R_{\text {eff,sel }}$ \\
\hline NGC 1380 & 0.44 & 0.54 & 0.75 & 0.80 \\
NGC 2434 & 0.07 & 0.06 & 0.19 & 0.11 \\
NGC 3115 & 0.82 & 1.00 & 0.88 & 1.00 \\
NGC 3379 & 0.53 & 0.77 & 0.78 & 0.90 \\
NGC 3585 & 0.57 & 0.90 & 0.73 & 0.90 \\
NGC 5846 & 0.72 & 0.78 & 0.86 & 0.90 \\
NGC 7192 & 0.29 & 0.83 & 0.50 & 0.80 \\
\hline
\end{tabular}

In the case of NGC 1380, a background galaxy cluster is placed right behind the galaxy and contaminates the candidate selection. Unfortunately, the HST photometry does not cover a large enough area to efficiently weed out resolved candidates. All other galaxies have very good success rates inside $1 R_{\text {eff }}$ typically between $\sim 80$ and $100 \%$ which validates the efficient candidate selection.

\subsection{Host galaxy masses}

The mass of a galaxy can be probed by its globular cluster system out to large radii ( $~ 22 R_{\text {eff }}$ ) including a significant fraction of the halo mass. In the past, several simple mass estimators based on the virial theorem, such as the projected mass estimator (Bahcall \& Tremaine 1981; Heisler et al. 1985), have been developed to derive masses of galaxy groups. Unfortunately, a key assumption of these mass estimators is that the tracer population follows the mass density of the probed potential. While to zeroth order this is true for galaxy groups, the assumption fails when globular clusters are used as tracers for galaxy potentials (see also Sect. 2.4).

Recently, a mass estimator was generalised to cases where the tracer population does not follow the mass profile (Evans et al. 2003). We use this tracer mass estimator to derive masses for our sample galaxies using radial velocities and projected radii of our globular cluster samples. For an isothermal potential $^{6}$, the general form of the estimator is

$M_{\text {press }}=\frac{C}{G N} \sum_{i}\left(v_{i, \mathrm{los}}-\langle v\rangle\right)^{2} R_{i}$

where

$C=\frac{16(\gamma-2 \beta)}{\pi(4-3 \beta)} \cdot \frac{4-\gamma}{3-\gamma} \cdot \frac{1-\left(r_{\text {in }} / r_{\text {out }}\right)^{3-\gamma}}{1-\left(r_{\text {in }} / r_{\text {out }}\right)^{4-\gamma}}$.

Here, $\langle v\rangle$ is the system's mean radial velocity and $\beta$ the anisotropy parameter $1-\sigma_{t}^{2} / \sigma_{r}^{2}$ which is unity for purely radial orbits and $-\infty$ for a system with solely tangential orbits

${ }^{6}$ A basic underlying assumption of all mass estimators is a steady state equilibrium. 
Table 7. Host galaxy masses in units of $10^{11} M_{\odot}$. The total mass was determined from the full set of globular clusters. Inner and outer projected radii which are defined by the projected radial spread of the sample are given in kpc. The rotational and pressure component of the total mass estimate are given separately. The expected uncertainty of the total mass estimate is $\sim 30-50 \%$. The last two columns show the total mass estimate inside $1 R_{\text {eff }}$ and the number of test particles. The galactocentric radius (in kpc) of the most distant globular cluster for the mass estimate inside one effective radius is given in column $R_{\text {eff,out }}$.

\begin{tabular}{lcccccccc}
\hline \hline Galaxy & $R_{\text {in }}$ & $R_{\text {out }}$ & $R_{\text {out },<1 \mathrm{R}_{\text {eff }}}$ & $M_{\text {rot }}$ & $M_{\text {press }}$ & $M_{\text {total }}$ & $M_{\text {total },<1 \mathrm{R}_{\text {eff }}}$ & $N_{\mathrm{GC},<1 \mathrm{R}_{\text {eff }}}$ \\
\hline NGC 1380 & 1.12 & 17.13 & 2.53 & 0.18 & 8.44 & 8.62 & 1.75 & 19 \\
NGC 2434 & 1.40 & 13.80 & 2.10 & 0.09 & 0.79 & 0.88 & $0.31^{a}$ & 5 \\
NGC 3115 & 1.33 & 14.55 & 4.82 & 0.26 & 2.93 & 3.19 & 0.70 & 18 \\
NGC 3379 & 0.84 & 10.41 & 2.88 & 0.09 & 2.76 & 2.85 & 0.96 & 10 \\
NGC 3585 & 2.26 & 19.14 & 2.73 & 0.21 & 2.62 & 2.83 & 1.41 & 9 \\
NGC 5846 & 2.31 & 24.65 & 1.63 & 0.27 & 11.6 & 11.9 & 6.38 & 18 \\
NGC 7192 & 3.95 & 38.02 & 3.62 & 1.21 & 4.47 & 5.68 & 0.89 & 5 \\
\hline
\end{tabular}

${ }^{a}$ No data are available inside $1 R_{\text {eff }}$; the given mass was calculated inside $2 R_{\text {eff }}$.

$b$ Masses were calculated using globular cluster data from our study and those of Kavelaars (1998) and Kuntschner et al. (2002).

(Binney 1981). The exponent of the three-dimensional density profile of the globular cluster population, defined through $\rho(r)=\rho_{0} \cdot r^{-\gamma}$, is not known a priori. However, to a good approximation the power-law rule $\gamma=1+\mathrm{d} \log \Sigma / \mathrm{d} \log R$ (Gebhardt et al. 1996) can be used to derive $\gamma$ from the surface density profiles in Sect. 2.4 assuming spherical symmetry. The projected radii $R_{\text {in }}$ and $R_{\text {out }}$ are taken as the 3-dimensional minimal and maximal galactocentric distances $r_{\text {in }}$ and $r_{\text {out }}$.

The mass estimator applies only to a pressure-supported tracer population. That is, any net rotation has to be subtracted from the sample before the tracer mass estimator is applied. We eliminate the mean rotational component by fitting a rotation curve to the entire globular cluster sample following Gebhardt et al. (2000). Total masses are calculated by adding the rotational component (assuming a flat rotation curve at large radii) to the pressure component from the tracer mass estimator

$M_{\mathrm{total}}=\frac{R_{\mathrm{out}}\left\langle v_{\mathrm{max}}\right\rangle^{2}}{G}+M_{\mathrm{press}}$

Total masses are calculated for isotropic globular cluster orbits $(\beta=0)$. For reasonably extreme anisotropies these mass estimates were found to vary by at most $\sim 30 \%$ (Evans et al. 2003). Taking into account the uncertainties in the mean system velocity and the rotational mass component as well as statistical fluctuations due to the limited sample size, we expect a $30-50 \%$ uncertainty in the total mass estimate. Table 7 summarizes the results for all sample galaxies.

\section{Line indices}

\subsection{Sampled luminosities}

The sampling of the globular cluster luminosity function (GCLF) is illustrated in Fig. 6. Our spectroscopic samples represent, strictly speaking, only small fractions of the entire globular cluster population and are biased towards high cluster masses. However, from the Milky Way and photometric studies, no large variations of globular cluster properties are expected with mass. Typical values of the sampled fraction of the entire GCLF down to the faintest cluster in the spectroscopic data set vary between 1.5 and $8.5 \%$. All spectroscopic subsamples are biased towards bright magnitudes and probe the bright end of the GCLF. Taking into account metallicity and age variations inside a globular cluster system our data will be biased towards young globular clusters if present.

Spectra of globular clusters with a total sampled luminosity of less than $\sim 10^{5} L_{\odot}$ are likely to be dominated by stochastic fluctuations of the number of bright stars (e.g. Renzini \& Fusi Pecci 1988; Renzini 1998; Puzia et al. 2002c). To convince ourselves that enough light is sampled by the slit we estimate the total luminosity $L_{\mathrm{T}}$ of our sample globular clusters from the photometry. We use the distance modulus of Tonry et al. (2001), foreground reddening maps of Schlegel et al. (1998), and bolometric corrections from Maraston (1998) in the equation

$L_{\mathrm{T}}=B C_{I} \cdot 10^{-0.4 \cdot\left(m_{I}-(m-M)-M_{\odot}-A_{I}\right)}$.

As $I$ band photometry is available for all globular cluster systems (see Table 2) we use $I$ magnitudes for our calculation. The absolute $I$ magnitude of the sun $\left(M_{I, \odot}=3.94\right)$ was taken from Cox (2000).

The lower right panel in Fig. 6 shows the distribution of luminosities for the entire globular cluster sample. The mean of the cluster luminosity distribution is $\langle\log L\rangle=5.85 \pm 0.03$ with a dispersion of $\sigma=0.37$ dex. Only one globular cluster has a total luminosity lower than $10^{5} L_{\odot}$ (GC\#10 in NGC 3379 with $L=7.2 \times 10^{4} L_{\odot}$, see also Table C.4). In other words, all clusters are far from the low-luminosity regime where the integrated light can be dominated by a few bright stars. Using the number-counts of Renzini (1998) we find that the total luminosity of each globular cluster is the integral over at least a few thousand stars.

The estimate mentioned above is based on photometry which measures all the light emitted by the cluster. The slits, however, sample less light depending on seeing conditions and mask alignment during the observations. Only four exposures, two NGC 5846 and two NGC 3585 frames, suffered during one night $(25 . / 26.5 .2001)$ from bad seeing $\left(\sim 1.5^{\prime \prime}\right)$. 

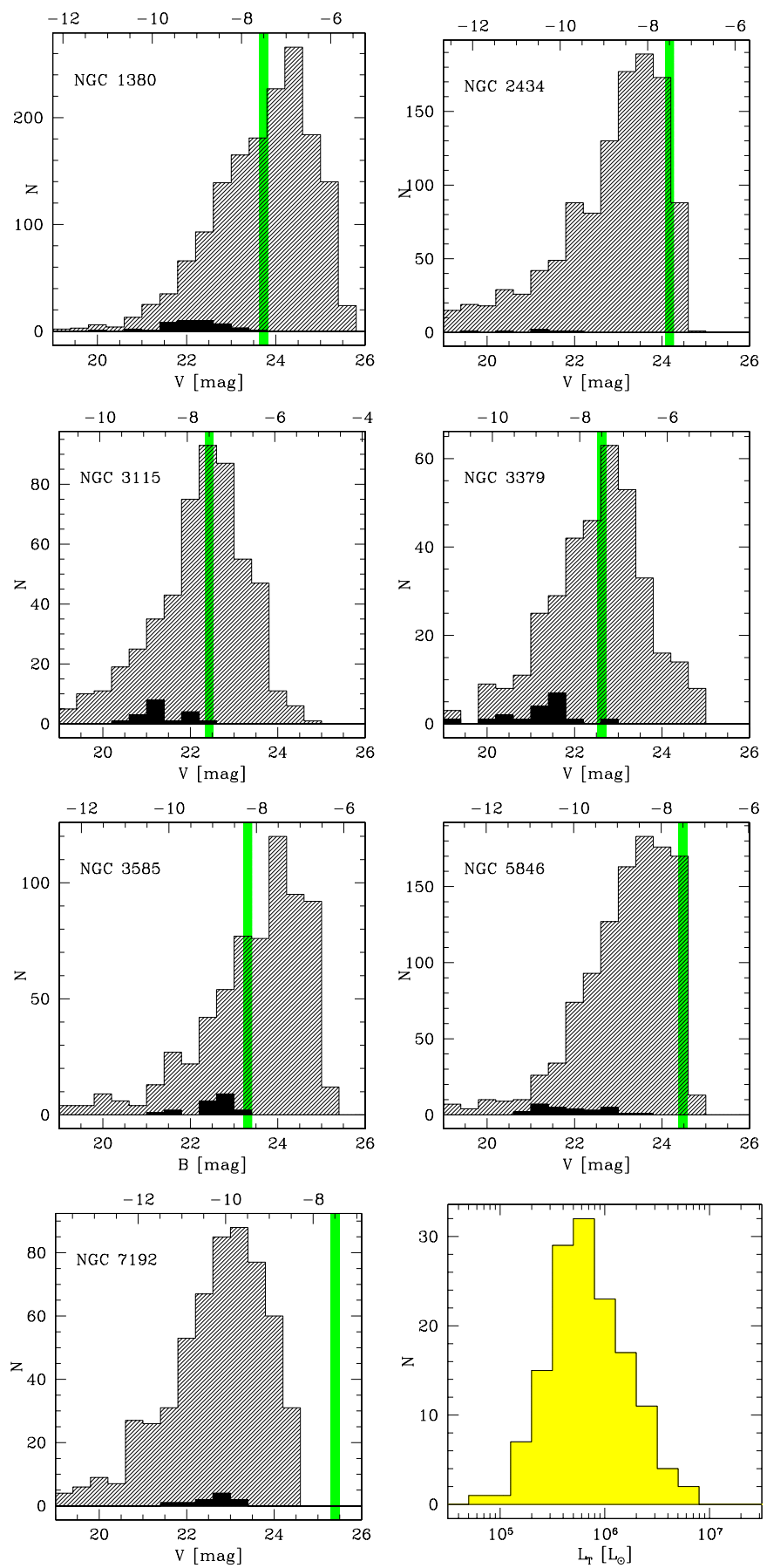

Fig. 6. Observed globular cluster luminosity functions (GCLFs). Hatched histograms show the magnitude distribution of the entire photometric sample. Solid histograms are spectroscopic samples. A vertical shaded band indicates the location of the GCLF turn over which is found in Local Group globular cluster systems and is expected at $M_{V} \approx-7.4$ to $-7.6 \mathrm{mag}$ (Harris 2001; Richtler 2003). Note that the panel for NGC 3585 refers to $B$ band magnitudes. An absolute magnitude scale is provided at the upper abscissa of each panel. Note that the GCLFs are not corrected for completeness. The lower right panel shows a histogram of globular cluster luminosities, $L_{\mathrm{T}}$, which are sampled by our spectroscopic data.

These exposures were assigned a low weighting factor during the combining process and do not affect the final spectrum.

\subsection{Calibration of lick line indices}

Lick indices (for passband definitions see Worthey et al. 1994; Worthey \& Ottaviani 1997) are measured on the fluxed and combined globular-cluster spectra. The wavelength range coverage allows for $17 \%$ of our sample to have $\mathrm{TiO}_{1}$ index measurements. The redder index $\mathrm{TiO}_{2}$ cannot be measured for all globular clusters. However, both indices are less instructive as they are affected by calibration uncertainties and stochastic fluctuation in the number of mostly contributing cool giant stars (Puzia et al. 2002c; Maraston et al. 2003; Thomas et al. 2003). Prior to performing the line-index measurements, the spectra were smoothed by a $\lambda$-dependent Gaussian kernel to match the Lick/IDS spectral resolution (Faber et al. 1985; Worthey \& Ottaviani 1997; Beasley et al. 2000; Puzia et al. 2002c). The transformation to the Lick system, the measurement of Lick indices, and the error analysis is performed in the same way as described in Puzia et al. (2002c).

In particular, the transformation to the Lick system was performed in the following way. During our observing runs in period 65, 66, and 67 we observed a total of 31 Lick standard stars which are used to accurately tune our spectroscopic system to the Lick/IDS characteristics. All standard-star spectra were observed with the same slit-size $\left(1.0^{\prime \prime}\right)$ and were extracted and smoothed in exactly the same way as the globular cluster spectra. By comparing our standard-star index measurements with published indices measured on original Lick/IDS spectra (Worthey et al. 1994; Worthey \& Ottaviani 1997) we calculate correction functions of the form

$I_{\mathrm{cal}}=I_{\mathrm{raw}}+\alpha$

where $I_{\text {cal }}$ and $I_{\text {raw }}$ are the calibrated and the measured indices, respectively. These functions allow us to reliably lock each index to the Lick system compensating for minor inaccuracies during the smoothing process and deviant continuumslopes, compared with original Lick spectra, due to our fluxcalibration. The comparison of selected indices between our measurements and the Lick systems is shown in Fig. 7. A major fraction of the scatter is due to the large errors of the Lick/IDS measurements which are about an order of magnitude larger than our standard-star values. Most indices require only a small correction while the calibration of very noisy indices, such as G4300 and Ca4455, remains uncertain. All globular-cluster indices are corrected with these zero-point offsets. Table 8 summarises the correction coefficients $\alpha$ used in Eq. (5) and the rms of the calibration.

Calibrated indices and their uncertainties for all globular clusters are presented in the Appendix B in Tables B.1 to B.9. A few index measurements are influenced by bad pixels inside the background and/or feature passband due to bad cosmic-ray interpolation. We discard these index measurements from our data. Particularly with regard to future age and metallicity determinations, an age resolution of $\sim 1-2$ Gyr requires Balmer line accuracies $\Delta \mathrm{H} \beta \lesssim 0.05 \AA$ and $\lesssim 0.1 \AA$ for the higher-order Balmer line indices, if age is considered as the only parameter which drives Balmer indices. This is not true in general because of the metallicity dependence of horizontal branch and turnoff temperatures (Maraston et al. 2003). We will take these 

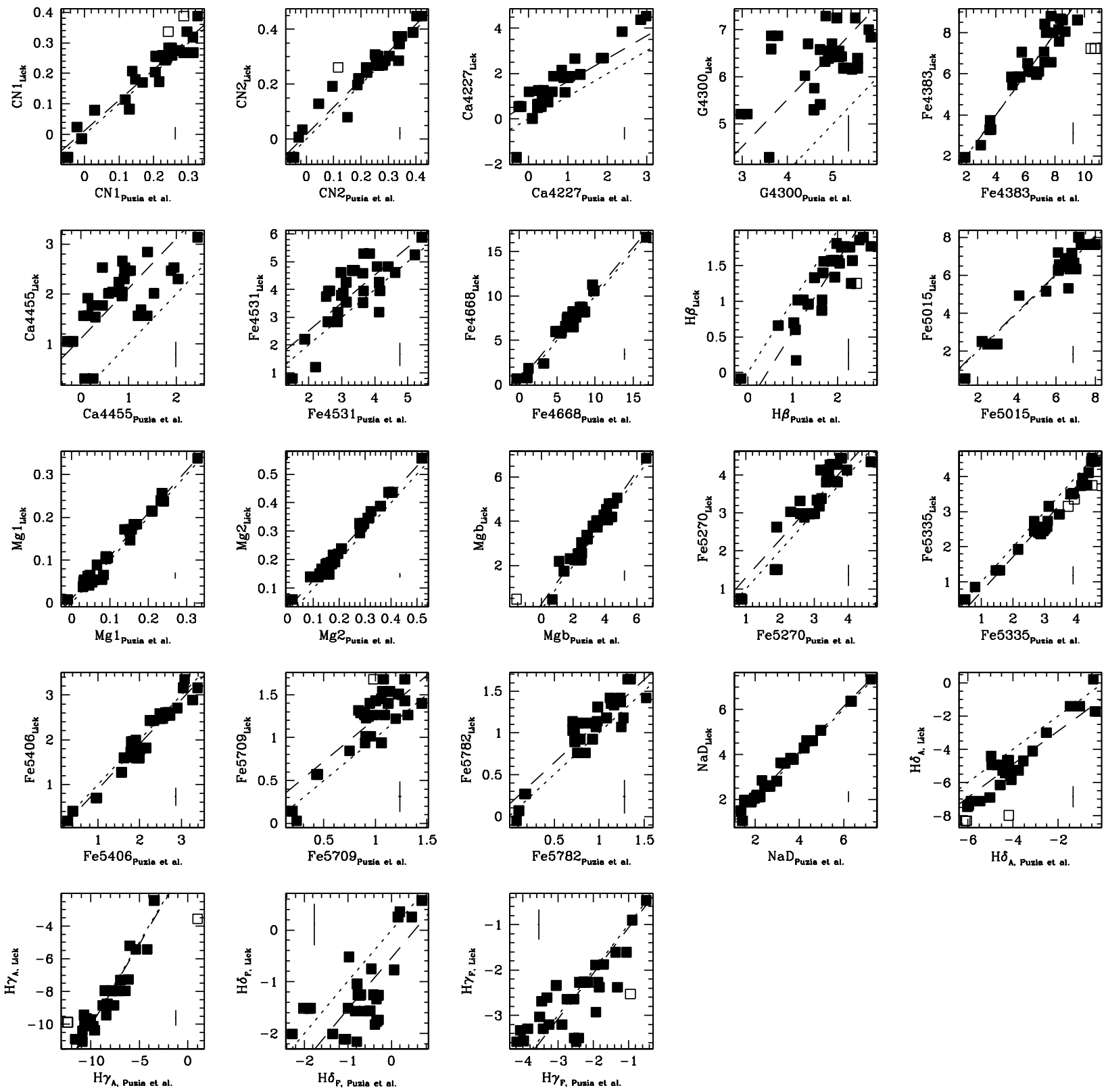

Fig. 7. Comparison of Lick index measurements on our smoothed standard-star spectra with the published Lick index values for corresponding stars taken from Worthey et al. (1994) and Worthey \& Ottaviani (1997). Dashed lines indicate the best least-square fit to the data using $\kappa-\sigma$ clipping. Dotted lines show the one-to-one relation. Data with exceptionally large errors or large deviations which were not used in the fitting process are shown as open squares. Note that offsets for $\mathrm{TiO}_{1}$ and $\mathrm{TiO}_{2}$ could not be determined due to the lack of wavelength coverage in our standard star spectra. Both these indices remain uncorrected. Typical error bars are indicated in a corner of each panel.

effects into account in future analyses (Puzia et al. in preparation). Very few objects achieve this high index accuracy. A metallicity resolution of 0.1 dex requires $\Delta[\mathrm{MgFe}]^{\prime} \lesssim 0.15 \AA^{7}$. About $40 \%$ of our data meets this criterion.

Clearly, the age resolution needs to be compromised for a large enough final sample size. Relaxed Balmer-index error cuts at $\Delta \mathrm{H} \beta=0.4 \AA$ and $0.6 \AA$ for the higher-order Balmer indices guarantee an age resolution of $\Delta t / t \approx 0.3$. An error cut at $\Delta[\mathrm{MgFe}]^{\prime}=0.2 \AA$ corresponds to a metallicity

\footnotetext{
${ }^{7}$ A good metallicity indicator is the composite index $[\mathrm{MgFe}]^{\prime}=$ $\sqrt{\mathrm{Mg} b \cdot(0.72 \mathrm{Fe} 5270+0.28 \mathrm{Fe} 5335)}$. It was designed to be formally independent of $[\alpha / \mathrm{Fe}]$ abundance ratio variations (Thomas et al. 2003).
}

resolution $\sim 0.25-0.4$ dex, depending on absolute metallicity. $\sim 50 \%$ of our sample comply with these selection criteria and allow detailed age/metallicity for individual globular clusters.

\subsection{Representative spectra}

Figure 8 shows representative spectra of NGC 5846 globular clusters of increasing $\mathrm{Mg} b$ index strength. The sequence shows nicely the anti-correlation between the strength of the Balmer line series and $\mathrm{Mg}$ and $\mathrm{Fe}$ metal lines from low to high metallicities. Note the influence of increasing metallicity on the continuum which lowers the continuum flux in the blue part of the 
Table 8. Summary of $\alpha$ coefficients and their rms. The corrections for the indices $\mathrm{TiO}_{1}$ and $\mathrm{TiO}_{2}$ could not be determined due to the lack of wavelength coverage in our standard-star spectra. Hence, both indices remain uncorrected.

\begin{tabular}{lrrc}
\hline \hline Index & zp $-\alpha$ & rms & Units \\
\hline $\mathrm{CN}_{1}$ & 0.013 & 0.032 & mag \\
$\mathrm{CN}_{2}$ & 0.017 & 0.035 & mag \\
$\mathrm{Ca} 4227$ & 0.664 & 0.587 & $\AA$ \\
$\mathrm{G} 4300$ & 1.517 & 0.745 & $\AA$ \\
$\mathrm{Fe} 4384$ & -0.019 & 0.637 & $\AA$ \\
$\mathrm{Ca} 4455$ & 1.112 & 0.554 & $\AA$ \\
$\mathrm{Fe} 4531$ & 0.521 & 0.716 & $\AA$ \\
$\mathrm{Fe} 4668$ & 0.471 & 0.594 & $\AA$ \\
$\mathrm{H} \beta$ & -0.435 & 0.279 & $\AA$ \\
$\mathrm{Fe} 5015$ & 0.061 & 0.515 & $\AA$ \\
$\mathrm{Mg} 1$ & 0.010 & 0.013 & $\mathrm{mag}$ \\
$\mathrm{Mg} 2$ & 0.028 & 0.014 & $\mathrm{mag}$ \\
$\mathrm{Mg} b$ & 0.234 & 0.304 & $\AA$ \\
$\mathrm{Fe} 5270$ & 0.295 & 0.370 & $\AA$ \\
$\mathrm{Fe} 5335$ & -0.281 & 0.197 & $\AA$ \\
$\mathrm{Fe} 5406$ & -0.101 & 0.187 & $\AA$ \\
$\mathrm{Fe} 5709$ & 0.223 & 0.208 & $\AA$ \\
$\mathrm{Fe} 5782$ & 0.140 & 0.157 & $\AA$ \\
$\mathrm{NaD}$ & 0.118 & 0.193 & $\AA$ \\
$\mathrm{TiO}$ & $\ldots$ & $\ldots$ & $\mathrm{mag}$ \\
$\mathrm{TiO}$ & $\ldots$ & $\ldots$ & $\mathrm{mag}$ \\
$\mathrm{H} \delta_{A}$ & -0.899 & 0.699 & $\AA$ \\
$\mathrm{H} \gamma_{A}$ & -0.098 & 0.773 & $\AA$ \\
$\mathrm{H} \delta_{F}$ & -0.539 & 0.609 & $\AA$ \\
$\mathrm{H} \gamma_{F}$ & -0.071 & 0.575 & $\AA$ \\
\hline & & &
\end{tabular}

spectrum. In general, most our spectra are of relatively high quality with an average $S / N$ of $\gtrsim 20$ per $\AA$ between 5000 and $5100 \AA$.

\section{Globular cluster data from the literature}

In the following, we collect published spectroscopic globularcluster data which will be used in future papers of this series. We focus only on high-quality line indices which were measured with the newer passband definitions of the Lick group (Worthey et al. 1994; Worthey \& Ottaviani 1997; Trager et al. 1998) and exclude index data measured with older passband definitions (Burstein et al. 1984).

Some data are measured with the new passband definitions of Trager et al. (1998). However, most Lick-index SSP model predictions are based the fitting functions of Worthey et al. (1994) and Worthey \& Ottaviani (1997). Their passband definitions differ for the indices $\mathrm{CN}_{1}, \mathrm{CN}_{2}, \mathrm{Ca} 4227, \mathrm{G} 4300, \mathrm{Fe} 4383$, $\mathrm{Ca} 4455, \mathrm{Fe} 4531, \mathrm{C}_{2} 4668$ (former Fe4668), Fe5709, Fe5782, $\mathrm{NaD}, \mathrm{TiO}_{1}$, and $\mathrm{TiO}_{2}$ from the passband definitions of Trager et al. (1998). To compare data in a homogeneous system, we calculate transformations to the Worthey passband system for indices which were measured with Trager et al. (1998) passband definitions. Table 9 summarises offsets for each index which are given in the sense

$\Delta I=I_{\operatorname{Tr} 98}-I_{\mathrm{Wo} 94}$
Table 9. Summary of index offsets between the Worthey et al. and Trager et al. passband system. Most offsets were calculated using our standard-star spectra and are given in the sense $\Delta I=I_{\mathrm{Tr} 98}-I_{\mathrm{Wo9} 4}$ with the rms of the transformation. Offsets for $\mathrm{TiO}_{1}$ were calculated using our globular cluster data since the standard star spectra do not cover the full wavelength range. $\mathrm{A} \mathrm{TiO}_{2}$ offset could not be determined due to the lack of wavelength coverage in both our standard-star and globular-cluster spectra.

\begin{tabular}{lrcc}
\hline \hline Index & $\Delta I$ & rms & Units \\
\hline $\mathrm{CN}_{1}$ & 0.010 & 0.025 & $\mathrm{mag}$ \\
$\mathrm{CN}_{2}$ & 0.014 & 0.020 & $\mathrm{mag}$ \\
$\mathrm{Ca} 4227$ & 0.330 & 0.297 & $\AA$ \\
$\mathrm{G} 4300$ & 0.936 & 0.692 & $\AA$ \\
$\mathrm{Fe} 4384$ & 0.166 & 0.501 & $\AA$ \\
$\mathrm{Ca} 4455$ & 0.509 & 0.225 & $\AA$ \\
$\mathrm{Fe} 4531$ & 0.334 & 0.127 & $\AA$ \\
$\mathrm{Fe} 4668$ & 0.211 & 0.175 & $\AA$ \\
$\mathrm{Fe} 5709$ & 0.211 & 0.251 & $\AA$ \\
$\mathrm{Fe} 5782$ & 0.166 & 0.177 & $\AA$ \\
$\mathrm{NaD}$ & 0.001 & 0.200 & $\AA$ \\
$\mathrm{TiO}_{1}$ & -0.003 & 0.017 & $\mathrm{mag}$ \\
\hline
\end{tabular}

Note that passband definitions for all Balmer line indices and the widely used $\mathrm{Mg}$ and $\mathrm{Fe}$ indices $\mathrm{Mg}_{2}, \mathrm{Mg} b, \mathrm{Fe} 5270$, and Fe5335 do not change between the two systems.

We consider only data which have sufficiently high $S / N$. A short description of each data set is given below. Age and metallicity estimates for each globular cluster sample are taken from the papers the data were published in.

\subsection{Elliptical galaxies}

NGC 1023 - Lick indices $\left(\mathrm{CN}_{2}, \mathrm{H} \beta, \mathrm{G} 4300, \mathrm{Ca} 4227, \mathrm{Fe} 5270\right.$, $\mathrm{Fe} 5335$, and $\mathrm{Mg}_{2}$ ) for 9 globular clusters were measured by Larsen \& Brodie (2002) with the passband definitions of Worthey et al. (1994) and Worthey \& Ottaviani (1997) on spectra taken with LRIS attached to the Keck I telescope. Some spectra have a $S / N$ on the lower limit to be useful for our future analyses. The sample spans a wide range of cluster ages with two very young globular clusters at $\sim 500 \mathrm{Myr}$ to objects at 15 Gyr. Metallicities range from $[\mathrm{Fe} / \mathrm{H}] \approx-2.0$ to solar values.

NGC 1399 - Forbes et al. (2001) measure a sub-set of Lick line indices $\left(\mathrm{H} \gamma_{A}, \mathrm{H} \beta, \mathrm{Mg}_{2}, \mathrm{Mg} b\right.$, and $\left.\langle\mathrm{Fe}\rangle\right)$ defined in Trager et al. (1998) and Worthey \& Ottaviani (1997) for 10 globular clusters on high- $S / N$ spectra taken with LRIS on Keck I. The majority of the sample are old ( $11 \mathrm{Gyr})$ globular clusters. Two clusters are likely to have intermediate ages around 2 Gyr. A broad range in metallicity is covered by the clusters with $[\mathrm{Fe} / \mathrm{H}]$ from $\sim-2.3$ to $\sim+0.4$ dex.

NGC 3610 - Eight globular clusters were observed by Strader et al. (2003a) using the LRIS instrument on the Keck I telescope. The relatively faint magnitudes of the clusters and the short exposure time resulted in medium- $S / N$ spectra on which Lick indices $\left(\mathrm{H} \delta_{A}, \mathrm{H} \gamma_{A}, \mathrm{H} \beta, \mathrm{CN}_{2}, \mathrm{Ca} 4227, \mathrm{G} 4300\right.$, $\mathrm{Fe} 5270, \mathrm{Fe} 5335, \mathrm{Mg}_{2}$, and $\mathrm{Mg} b$ ) were measured using 


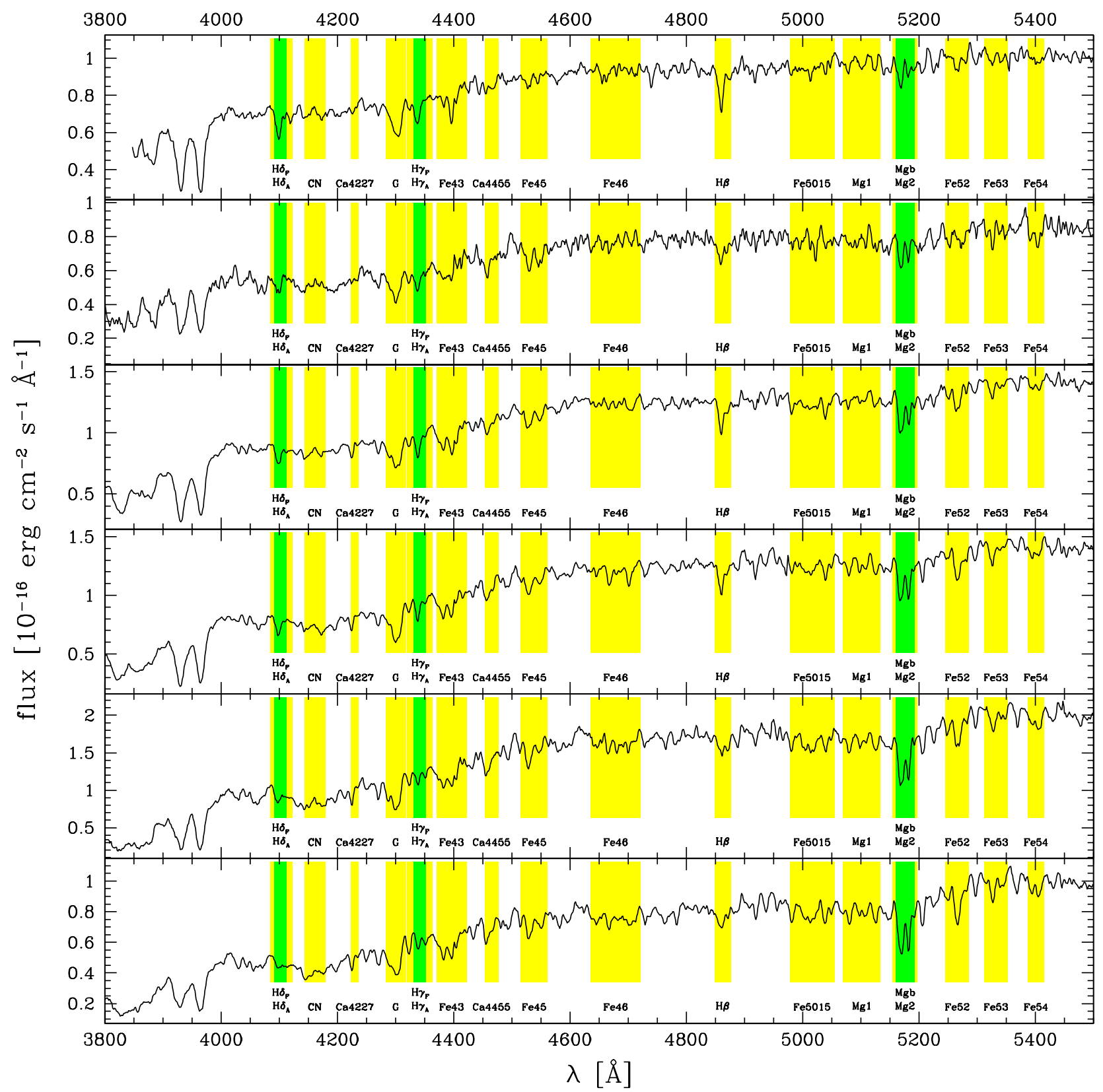

Fig. 8. Representative spectra from our final globular cluster sample. The relative $\mathrm{Mg} b$ index strength increases from the upper to the lower panel. Note the anti-correlation in the strength of some prominent spectral features such as Balmer lines and the $\mathrm{Mg} b$ feature at $\sim 5180 \AA$. All spectra are taken from the sample of globular clusters in NGC 5846 to demonstrate the influence of increasing metallicity on the continuum flux in the blue (note the changing ordinate scale). Feature passbands of measured Lick indices are shaded and labeled accordingly. Where two index passbands overlap the narrower is shaded darker and the label is elevated.

passband definitions of Trager et al. (1998) and Worthey \& Ottaviani (1997). Except for one intermediate-age globular cluster ( $\sim 3 \mathrm{Gyr})$ with a super-solar metallicity the entire sample appears old covering a wide metallicity range from $[\mathrm{Fe} / \mathrm{H}] \approx-2.3$ to 0.0 dex.

$N G C 4365$ - Larsen et al. (2003) measure a subset of Lick indices $\left(\mathrm{H} \beta, \mathrm{H} \gamma_{A}, \mathrm{H} \delta_{A}, \mathrm{Fe} 5270, \mathrm{Fe} 5335, \mathrm{Mg}_{2}\right.$, and $\left.\mathrm{Mg} b\right)$ for 14 globular clusters nine of which are likely to be intermediate-age metal-rich objects $(-0.4 \lesssim[\mathrm{Z} / \mathrm{H}] \lesssim 0.0$, 2-5 Gyr). The remaining clusters are consistent with old (10-15 Gyr) stellar populations covering a wide range in metallicity $(-2.5 \lessgtr[\mathrm{Z} / \mathrm{H}] \lessgtr 0.0)$. All indices were measured using passband definitions of Worthey et al. (1994) and Worthey \& Ottaviani (1997). The data were taken with LRIS attached to the Keck I telescope.

\subsection{Lenticular/SO galaxies}

NGC 3115 - High-quality spectra of 17 globular clusters have been taken by Kuntschner et al. (2002) with the FORS2 instrument at the VLT. The full set of 25 Lick line indices was measured with passband definitions of Worthey \& Ottaviani (1997) and Trager et al. (1998). The authors 

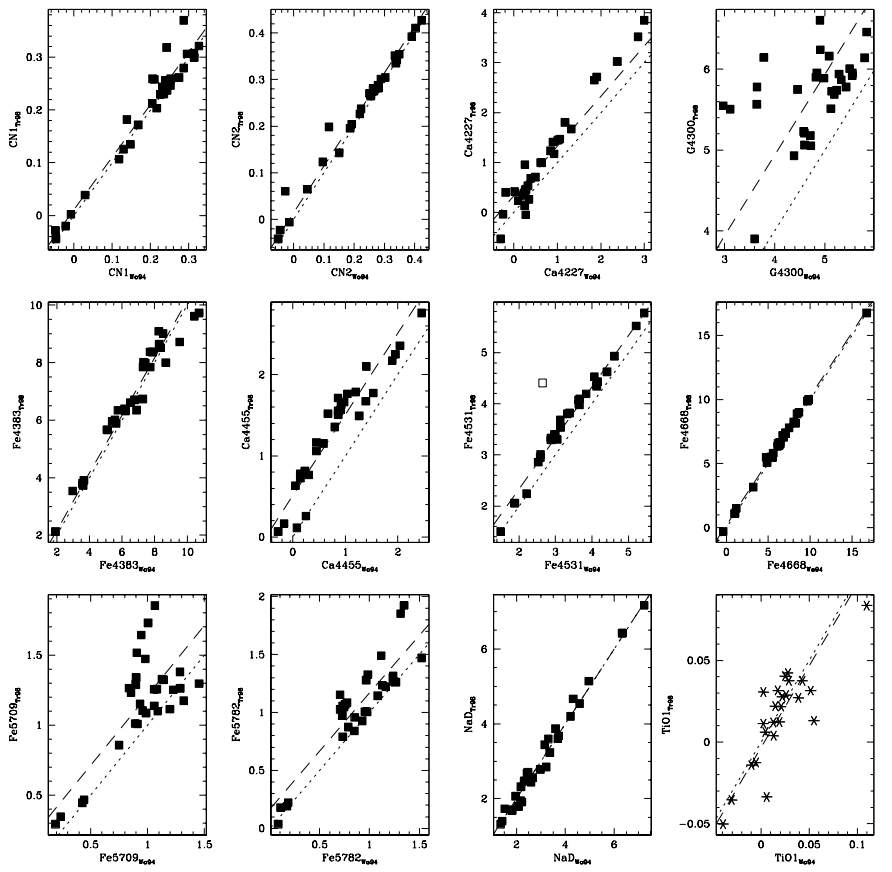

Fig. 9. Comparison of Lick index measurements performed with Worthey et al. and Trager et al. passband definitions for which passband definitions differ between the two systems. Spectra of Lick standard stars were used, except for $\mathrm{TiO}_{1}$ where we used globular cluster data. Offsets between the two systems are summarised in Table 9.

find a coeval old $(\sim 12 \mathrm{Gyr})$ set of globular clusters which covers a wide range in metallicity from -1.5 dex up to solar values.

$N G C 4594$ - 14 globular cluster spectra of medium $S / N$ have been obtained by Larsen et al. (2002a) with the LRIS on the Keck I telescope. Lick indices $\left(\mathrm{H} \delta_{A}, \mathrm{H} \gamma_{A}, \mathrm{H} \beta, \mathrm{Fe} 5270\right.$, $\mathrm{Fe} 5335, \mathrm{Mg}_{2}$, and $\mathrm{Mg} b$ ) using Worthey et al. (1994) and Worthey \& Ottaviani (1997) passband definitions were measured. The sample contains globular clusters with ages between 10 and 15 Gyr and a large metallicity spread from very metal-poor to super-solar abundance clusters.

\subsection{Late-type galaxies}

Milky Way - The full set of 25 Lick indices was measured on high-quality spectra for 12 galactic globular clusters by Puzia et al. (2002c) using passband definitions of Worthey et al. (1994), Worthey \& Ottaviani (1997), and Trager et al. (1998). The data were obtained with the Boller \& Chivens Spectrograph of ESO's $1.52 \mathrm{~m}$ on La Silla. As the Milky Way globular clusters consist of old stellar populations all sample clusters have ages in the range 10-15 Gyr. Their metallicities range from $[\mathrm{Fe} / \mathrm{H}]=-1.48$ to -0.17 dex. This sample is augmented by the data set of Trager et al. (1998) which adds 12 old metal-poor globular clusters with metallicities from $[\mathrm{Fe} / \mathrm{H}]=-2.29$ up to -0.73 dex. The Trager et al. data provide line indices for 21 passbands defined in their own work. Index measurements for higher-order Balmer line indices which are defined by Worthey \& Ottaviani (1997) are documented in
Kuntschner et al. (2002) and are added to the Trager et al. data set. Where the Puzia et al. and the Trager et al./Kuntschner et al. data set have objects in common we prefer data of Puzia et al. (2002c) over the other two because of systematically smaller uncertainties.

M31 - Trager et al. (1998) measure Lick line indices defined in the same work for 18 globular clusters. Index measurements for higher-order Balmer lines are provided by Kuntschner et al. (2002) and added to the former data.

$M 33$ - The Lick indices $\mathrm{G} 4300, \mathrm{H} \beta, \mathrm{Mg}_{2}, \mathrm{Fe} 5270$, and Fe5335 were measured by Chandar et al. (2002) with the passband definitions of Worthey et al. (1994) for 21 globular clusters. The sample clusters have metallicities from $[\mathrm{Fe} / \mathrm{H}]=-2.0$ to -0.5 dex and ages from a few Gyr to $\sim 15$ Gyr. The data were taken with the HYDRA multifiber spectrograph at WIYN $3.5 \mathrm{~m}$ telescope (KPNO).

M81 - Schroder et al. (2002) measure Lick indices with passbands defined in Trager et al. (1998) for 16 globular cluster candidates. Their data were obtained with the LRIS instrument. Most of the objects are consistent with stellar populations spanning ages between 8 and $17 \mathrm{Gyr}$ and metallicities between $[\mathrm{Fe} / \mathrm{H}]=-2.3$ and solar values. One globular cluster candidate appears to be of intermediate age $(\sim 3 \mathrm{Gyr})$ and intermediate metallicity $([\mathrm{Fe} / \mathrm{H}] \approx$ $-1.0)$. As M 81 has a negative systemic radial velocity $\left(-34 \pm 4 \mathrm{~km} \mathrm{~s}^{-1}\right.$ de Vaucouleurs et al. 1991) the selection of globular clusters remains rather uncertain. Schroder et al. (2002) reduce the ambiguities by restricting the sample to objects with small projected radii. Furthermore, they compare the strength of $\mathrm{Ca} \mathrm{I}$ and $\mathrm{H} \delta$ absorption lines with photometric colours and exclude stars using the technique of Perelmuter et al. (1995).

$L M C$ - Lick indices of 24 globular clusters have been measured on high- $S / N$ spectra by Beasley et al. (2002). The authors use passband definitions of Trager et al. (1998) to measure their 16 bluest indices $\left(\mathrm{CN}_{1}\right.$ to $\left.\mathrm{Fe} 5406\right)$ and definitions of Worthey \& Ottaviani (1997) to measure 4 higherorder Balmer line indices. Their sample spans metallicities from $[\mathrm{Fe} / \mathrm{H}]=-2.1$ up to solar values with globular cluster ages of a few million years up to old objects of $\sim 15 \mathrm{Gyr}$. The observations were performed with the FLAIR instrument at the $1.2 \mathrm{~m}$ UK Schmidt telescope (AAO).

Fornax - Strader et al. (2003b) provide a sub-set of Lick index measurements $\left(\mathrm{CN}_{1}, \mathrm{CN}_{2}, \mathrm{Ca} 4227, \mathrm{G} 4300, \mathrm{H} \beta, \mathrm{Mg}_{2}, \mathrm{Mg} b\right.$, $\mathrm{Fe} 5270, \mathrm{Fe} 5335, \mathrm{H} \gamma_{A}$, and $\mathrm{H} \delta_{A}$ ) with passbands defined by Trager et al. (1998) and Worthey \& Ottaviani (1997) for 4 globular clusters in the Fornax dwarf galaxy. The clusters appear to be metal-poor $([\mathrm{Fe} / \mathrm{H}] \approx-1.8)$ and old $(\sim 15 \mathrm{Gyr})$ with one cluster being younger by $\sim 2-3$ Gyr. The objects were observed with the LRIS instrument.

\section{Summary}

We present a homogeneous set of Lick indices for 143 extragalactic globular clusters in seven early-type galaxies located in different environments. The indices were measured on highquality VLT spectra and are currently the largest homogeneous spectroscopic data set of extragalactic globular cluster systems. 
The candidate pre-selection for follow-up spectroscopy was confirmed to work very efficiently. Inside one effective radius the success rates are between $\sim 80-100 \%$ for galaxies located at high galactic latitudes $\left(|b| \gtrsim 40^{\circ}\right)$.

We provide a method to reduce the number of contaminating fore- and background objects during the candidate selection. A combination of near-infrared and optical colours in a $I-K$ vs. $B-K$ colour-colour diagram allows to disentangle foreground stars and background galaxies from the globular cluster population very efficiently. Fractional contamination can be reduced to $\$ 10 \%$.

We fit surface brightness and surface density profiles to the galaxy light and the globular cluster system and find that globular cluster systems have in general comparable or more extended profiles than the galaxy light. By dividing the clearly multi-modal globular cluster populations in blue and red subsamples, we find that both have similar profile slopes. A bruteforce division of the remaining single-peak systems reveals that the red globular cluster sub-population is more concentrated than its blue counterpart. This interesting point will be further analysed in a future paper.

Using the radial velocity information of our globular cluster samples we measure dynamical masses for the seven host galaxies which have total masses between $\sim 8.8 \times 10^{10} M_{\odot}$ and $\sim 1.2 \times 10^{12} M_{\odot}$.

The accuracy of index measurements allows an age resolution $\Delta t / t \approx 0.3$ and a metallicity resolution in the range $\sim 0.25-0.4$ dex depending on the absolute metallicity. Hence, $\sim 50 \%$ of our data allows detailed age/metallicity determinations for individual globular clusters.

Acknowledgements. We thank Rupali Chandar for providing an electronic list of her globular cluster line index measurements and Karl Gebhardt for helping with his analysis software. THP gratefully acknowledges the support by the German Deutsche Forschungsgemeinschaft, $D F G$ project number Be 1091/10-2.

\section{References}

Ashman, K. M., \& Zepf, S. E. 1992, ApJ, 384, 50

Ashman, K. A., Bird, C. M., \& Zepf, S. E. 1994, AJ, 108, 2348

Ashman, K. M., \& Zepf, S. E. 1998, Globular Cluster Systems (Cambridge University Press)

Bahcall, J. N., \& Tremaine, S. 1981, ApJ, 244, 805

Barmby, P., Huchra, J. P., Brodie, J. P., et al. 2000, AJ, 119, 727

Beasley, M. A., Sharples, R. M., Bridges, T. J., et al. 2000, MNRAS, 318,1249

Beasley, M. A., Hoyle, F., \& Sharples, R. M. 2002, MNRAS, 336, 168

Bertin, E., \& Arnouts, S. 1996, A\&AS, 117, 393

Binney, J. 1981, Structure and Evolution of Normal Galaxies, ed. D. Lynden-Bell \& S. M. Fall (Cambridge: Cambridge University Press)

Binney, J., \& Merrifield, M. 1998, Galactic Astronomy (Princeton University Press)

Bothun, G. D., \& Gregg, M. D. 1990, ApJ, 350, 73

Burstein, D., Faber, S. M., Gaskell, C. M., \& Krumm, N. 1984, ApJ, 287, 586

Buta, R., \& Williams, K. L. 1995, AJ, 109, 543

Chandar, R., Bianchi, L., Ford, H. C., \& Sarajedini, A. 2002, ApJ, 564, 712
Cohen, J. G., Blakeslee, J. P., \& Ryzhov, A. 1998, ApJ, 496, 808

Cote, P., Marzke, R. O., \& West, M. J. 1998, ApJ, 501, 554

Cox, A. N. 2000, in Allen's Astrophysical Quantities, 4th ed., ed. A. N. Cox (Springer)

Evans, N. W., Wilkinson, M. I., Perrett, K. M., \& Bridges, T. J. 2003, ApJ, 583, 752

Faber, S. M., Friel, E. D., Burstein, D., \& Gaskell, C. M. 1985, ApJS, 57, 711

Faber, S. M. 1972, A\&A, 20, 361

Forbes, D. A., Beasley, M. A., Brodie, J. P., \& Kissler-Patig, M. 2001, ApJ, 563, L143

Gabasch, et al. 2004, in preparation

Gebhardt, K., et al. 1996, AJ, 112, 105

Gebhardt, K., \& Kissler-Patig, M. 1999, AJ, 118, 1526

Gebhardt, K., Pryor, C., O'Connell, R. D., Williams, T. B., \& Hesser, J. E. 2000, AJ, 119, 1268

Gnedin, O. Y., \& Ostriker, J. P. 1997, ApJ, 474, 223

González, J. J. 1993, Ph.D. Thesis, University of California, Santa Cruz

Goudfrooij, P., Mack, J., Kissler-Patig, M., Meylan, G., \& Minniti, D. 2001, MNRAS, 322, 643

Gössl, C. A., \& Riffeser, A. 2002, A\&A, 381, 1095

Guzman, R., Jangren, A., Koo, D. C., Bershady, M. A., \& Simard, L. 1998, ApJ, 495, L13

Harris, W. E., \& van den Bergh, S. 1981, AJ, 86, 1627

Harris, W. E. 1996, AJ, 112, 1487, for the 1999 update see http://physun.physics.mcmaster.ca/ ${ }^{\sim}$ harris/mwgc. dat

Harris, W. E. 2001, in Saas-Fee Advanced School on Star Clusters, ed. L. Labhardt \& B. Binggeli (course 28) (New York: Springer)

Heidt, J., Appenzeller, I., Gabasch, A., et al. 2003, A\&A, 398, 49

Heisler, J., Tremaine, S., \& Bahcall, J. N. 1985, ApJ, 298, 8

Hempel, M., Hilker, M., Kissler-Patig, M., et al. 2003, A\&A, 405, 487

Holtzman, J. A., Burrows, C. J., Casertano, S., et al. 1995a, PASP, 107, 1065

Holtzman, J. A., Hester, J. J., Casertano, S., et al. 1995b, PASP, 107, 156

Horne, K. 1986, PASP, 98, 609

Huchra, J., Stauffer, J., \& van Speybroeck, L. 1982, ApJ, 259, L57

Jordán, A., Côté, P., West, M. J., \& Marzke, R. O. 2002, ApJ, 576, L113

Kavelaars, J. J. 1998, Ph.D. Thesis

Kinney, A. L., Calzetti, D., Bohlin, R. C., et al. 1996, ApJ, 467, 38

Kissler-Patig, M., Richtler, T., Storm, J., \& della Valle, M. 1997, A\&A, 327, 503

Kissler-Patig, M., Brodie, J. P., Schroder, L. L., et al. 1998, AJ, 115, 105

Kissler-Patig, M. 2000, Rev. Mod. Astron., 13, 13

Kissler-Patig, M., Brodie, J. P., \& Minniti, D. 2002, A\&A, 391, 441

Kormendy, J., \& Djorgovski, S. 1989, ARA\&A, 27, 235

Kundu, A., \& Whitmore, B. C. 1998, AJ, 116, 2841

Kundu, A., \& Whitmore, B. C. 2001a, AJ, 121, 2950

Kundu, A., \& Whitmore, B. C. 2001b, AJ, 122, 1251

Kuntschner, H., Ziegler, B. L., Sharples, R. M., Worthey, G., \& Fricke, K. J. 2002, A\&A, 395, 761

Larsen, S. S., Brodie, J. P., Huchra, J. P., Forbes, D. A., \& Grillmair, C. J. 2001, AJ, 121, 2974

Larsen, S. S., \& Brodie, J. P. 2002, AJ, 123, 1488

Larsen, S. S., Brodie, J. P., Beasley, M. A., \& Forbes, D. A. 2002, AJ, 124,828

Larsen, S. S., Brodie, J. P., Beasley, M. A., et al. 2003, ApJ, 585, 767

Mannucci, F., Basile, F., Poggianti, B. M., et al. 2001, MNRAS, 326 745

Maraston, C. 1998, MNRAS, 300, 872 
Maraston, C., Kissler-Patig, M., Brodie, J. P., Barmby, P., \& Huchra, J. P. 2001, A\&A, 370, 176

Maraston, C., Greggio, L., Renzini, A., et al. 2003, A\&A, 400, 823

McElroy, D. B. 1995, ApJS, 100, 105

O'Connell, R. W. 1976, ApJ, 206, 370

Pahre, M. A. 1999, ApJS, 124, 127

Perelmuter, J., Brodie, J. P., \& Huchra, J. P. 1995, AJ, 110, 620

Puzia, T. H., Kissler-Patig, M., Brodie, J. P., \& Huchra, J. P. 1999, AJ, 118,2734

Puzia, T. H., Zepf, S. E., Kissler-Patig, M., et al. 2002a, A\&A, 391, 453

Puzia, T. H., Kissler-Patig, M., Brodie, J. P., et al. 2002b, IAU Symp., 207, ed. D. Geisler, E. K. Grebel, \& D. Minniti

Puzia, T. H., Saglia, R. P., Kissler-Patig, M., et al. 2002c, A\&A, 395, 45

Reader, J., \& Corliss, Ch. H. 1981, CRC Handbook of Chemistry and Physics

Renzini, A. 1998, AJ, 115, 2459

Renzini, A., \& Fusi Pecci, F. 1988, ARA\&A, 26, 199

Richtler, T. 2003, in Stellar Candles for the Extragalactic Distance Scale, Concepcion, Dec. 2002, Lecture Notes in Physics (Springer), 635,281

Robin, A. C., Haywood, M., Crézé, M., Ojha, D. K., \& Bienaymé, O. 1996, A\&A, 305, 125

Schlegel, D. J., Finkbeiner, D. P., \& Davis, M. 1998, ApJ, 500, 525

Schroder, L. L., Brodie, J. P., Kissler-Patig, M., Huchra, J. P., \& Phillips, A. C. 2002, AJ, 123, 2473

Schweizer, F., Miller, B. W., Whitmore, B. C., \& Fall, S. M. 1996, AJ, 112,1839
Silverman, B. W. 1986, Density Estimation for Statistics and Data Analysis, Chap and Hall/CRC Press, Inc.

Soifer, B. T., Neugebauer, G., Matthews, K., et al. 2001, AJ, 122, 1213

Strader, J., Brodie, J. P., Schweizer, F., Larsen, S. S., \& Seitzer, P. 2003a, AJ, 125, 626

Strader, J., Brodie, J. P., Forbes, D. A., Beasley, M. A., \& Huchra, J. P. 2003b, AJ, 125, 1291

Thomas, D., Greggio, L., \& Bender, R. 1998, MNRAS, 296, 119

Thomas, D., Maraston, C., \& Bender, R. 2003, MNRAS, 339, 897

Tinsley, B. M. 1979, ApJ, 229, 1046

Tonry, J. L., Dressler, A., Blakeslee, J. P., et al. 2001, ApJ, 546, 681

Trager, S. C., Worthey, G., Faber, S. M., Burstein, D., \& Gonzalez, J. J. 1998, ApJS, 116, 1

Tripicco, M. J., \& Bell, R. A. 1995, AJ, 110, 3035

Tsujimoto, T., Nomoto, K., Yoshii, Y., et al. 1995, MNRAS, 277, 945

Tully, R. B. 1988, Nearby Galaxies Catalog (Cambridge and New York: Cambridge University Press)

van de Kamp, P. 1967, Principles of Astrometry (San Francisco: W. H. Freeman)

de Vaucouleurs, G., de Vaucouleurs, A., Corwin, H. G. Jr., et al. 1991, Third Reference Catalogue of bright galaxies (Berlin: Springer)

Whitmore, B. C., Miller, B. W., Schweizer, F., \& Fall, S. M. 1997, AJ, 114,1797

Woosley, S. E., \& Weaver, T. A. 1995, ApJS, 101, 181

Worthey, G., Faber, S. M., \& Gonzalez, J. J. 1992, ApJ, 398, 69

Worthey, G., Faber, S. M., Gonzalez, J. J., \& Burstein, D. 1994, ApJS, 94, 687

Worthey, G., \& Ottaviani, D. L. 1997, ApJS, 111, 377 
T. H. Puzia et al.: VLT spectroscopy of globular cluster systems. I., Online Material $p 1$

\section{Online Material}




\section{Appendix A: Spectra of objects \#10 and \#15 in the NGC 3115 globular cluster sample}

Spectra of stars and globular clusters can partly be disentangled by means of relative line strengths of spectral features such as Balmer lines and Ca I (4227 ̊) (Perelmuter et al. 1995). In globular cluster spectra the intensity of Balmer lines dominates in general the strength of the $\mathrm{Ca}$ I feature. This ratio approaches unity at high metallicities. On the other hand, dwarfs later than $\sim \mathrm{G} 3 \mathrm{~V}$ show a strong $\mathrm{Ca}$ I feature relative to Balmer lines. This is illustrated in Fig. A.1 where the spectra of objects \#10 and \#15 are compared with an average globular cluster spectrum (using bona-fide globular clusters in NGC 5846) and two stellar spectra of a late-type dwarf (K5 V) and a cool giant (K3 III).

The Ca I feature is not detectable in both object spectra (\#10 and \#15). However, judging by the relative strengths of the $\mathrm{Mg} b$ feature and the Balmer line $\mathrm{H} \beta$ both spectra are closer to the mean globular cluster spectrum than to both stellar spectra. This is less obvious for object \#15 than for the spectrum of object \#10.
The two objects have rather red colours: $I-K=1.02$ and $B-K=2.62$ for \#10, and $I-K=2.04$ and $B-K=4.40$ for \#15. The colours of object \#10 are consistent with late-type $\mathrm{G}$ dwarfs. Object \#15, on the other hand, is too red for its spectrum to be a dwarf. In this case strong molecular absorption bands would be detectable which is not the case. Its colours in combination with the type of the spectrum are rather consistent with a K giant (Cox 2000).

As an additional test we cross-correlate both object spectra with the two stellar spectra and the mean globular cluster spectrum and use the height of the cross-correlation peak (CCP) as a measure of similarity. It is important to note that the two stellar spectra and the mean globular cluster spectrum, which are considered as templates, have similarly high $S / N$ values. For object \#10, the test yields the highest CCP for the globular cluster spectrum. The spectra of object \#15 and the K giant are most alike by means of this test. Both results are, however, not significantly different from the cross-correlations with the remaining template spectra. 
T. H. Puzia et al.: VLT spectroscopy of globular cluster systems. I., Online Material $p 3$

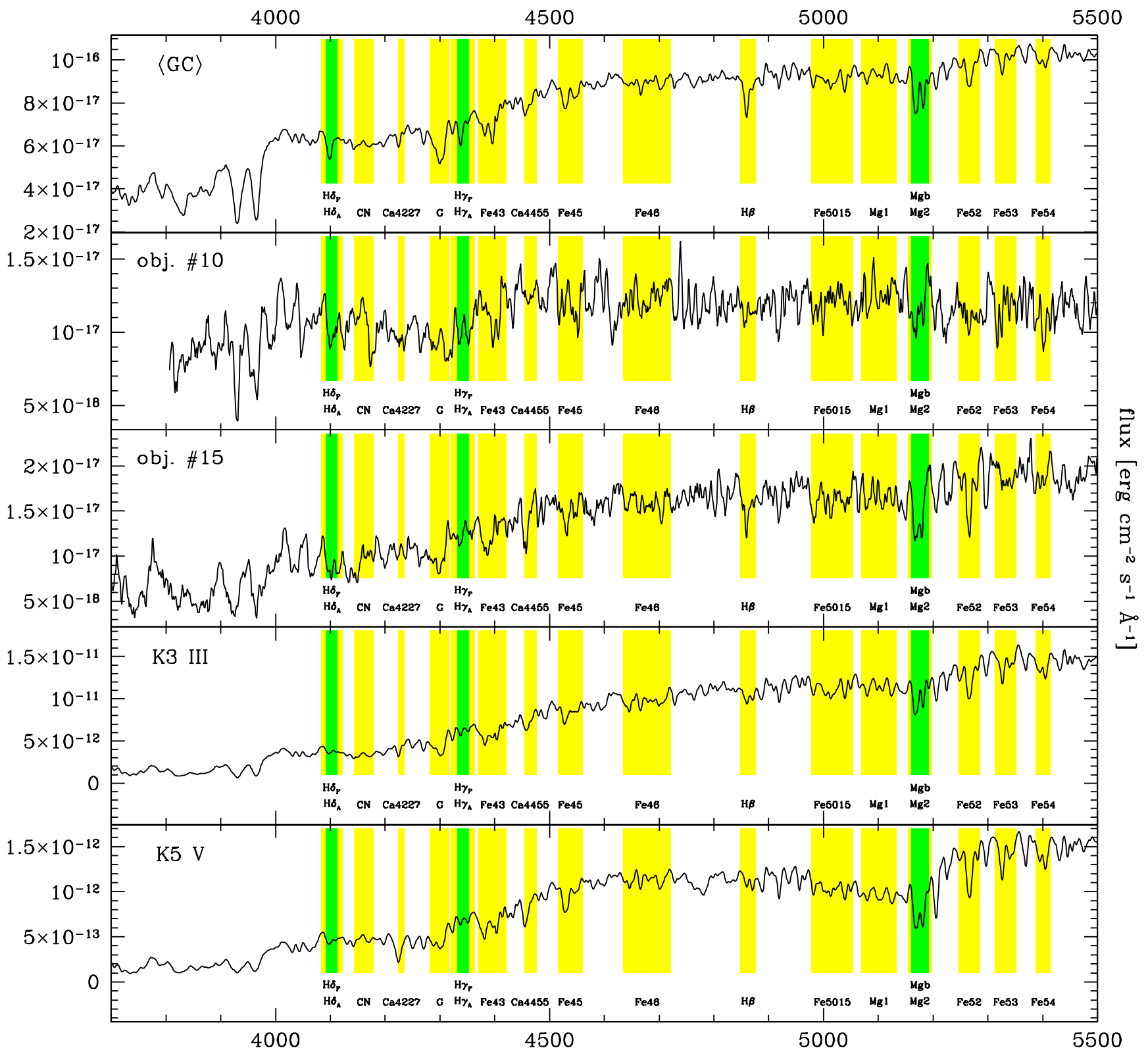

Fig. A.1. Spectra from top to bottom: mean globular cluster spectrum of NGC 5846 globular clusters, object \#10 and \#15 in NGC 3115 , a $\mathrm{K} 3$ giant, and a K5 dwarf spectrum. The latter two spectra were taken from our sample of Lick standard stars. All spectra are in rest frame and were smoothed to the Lick system resolution. Light shaded regions indicate feature passbands of Lick indices. Dark shaded regions indicate narrow passbands which overlap with broader passbands. The label for the narrow index is elevated. 